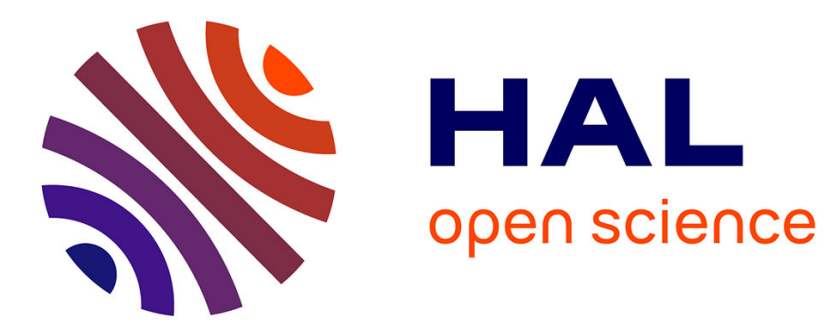

\title{
The Internal Structure of Mercury's Core Inferred From Magnetic Observations
}

\author{
I. Wardinski, Hagay Amit, B. Langlais, E. Thébault
}

\section{To cite this version:}

I. Wardinski, Hagay Amit, B. Langlais, E. Thébault. The Internal Structure of Mercury's Core Inferred From Magnetic Observations. Journal of Geophysical Research. Planets, 2021, 126 (12), 10.1029/2020JE006792 . hal-03503852

\section{HAL Id: hal-03503852 https://hal.science/hal-03503852}

Submitted on 28 Dec 2021

HAL is a multi-disciplinary open access archive for the deposit and dissemination of scientific research documents, whether they are published or not. The documents may come from teaching and research institutions in France or abroad, or from public or private research centers.
L'archive ouverte pluridisciplinaire HAL, est destinée au dépôt et à la diffusion de documents scientifiques de niveau recherche, publiés ou non, émanant des établissements d'enseignement et de recherche français ou étrangers, des laboratoires publics ou privés.

\section{(1)(1) $\$(0)$}

Distributed under a Creative Commons Attribution - NonCommercial - ShareAlikel 4.0 


\section{JGR Planets}

\section{RESEARCH ARTICLE 10.1029/2020JE006792}

Key Points:

- We model Mercury's internal magnetic field from MESSENGER data with spherical harmonics

- Our core field model contains nonaxisymmetric features from which we make inferences of Mercury's internal structure

- We estimate Mercury's inner core radius of $\sim 500-660 \mathrm{~km}$ and a corresponding thickness of a top stratified layer of $\sim 880-500 \mathrm{~km}$

Correspondence to:

I. Wardinski,

wardinski@unistra.fr

Citation:

Wardinski, I., Amit, H., Langlais, B., \& Thébault, E. (2021). The internal structure of Mercury's core inferred from magnetic observations. Journal of Geophysical Research: Planets, 126, e2020JE006792. https://doi.org/10.1029/2020JE006792

Received 5 DEC 2020 Accepted 1 DEC 2021

Author Contributions: Conceptualization: I. Wardinski Formal analysis: I. Wardinski Funding acquisition: B. Langlais Investigation: I. Wardinski, H. Amit Methodology: H. Amit

Resources: I. Wardinski

Software: I. Wardinski

Validation: B. Langlais

Writing - original draft: I. Wardinski,

H. Amit, B. Langlais

Writing - review \& editing: I

Wardinski, H. Amit, B. Langlais

(C) 2021. The Authors.

This is an open access article under the terms of the Creative Commons Attribution-NonCommercial-NoDerivs License, which permits use and distribution in any medium, provided the original work is properly cited, the use is non-commercial and no modifications or adaptations are made.

\section{The Internal Structure of Mercury's Core Inferred From Magnetic Observations}

\author{
I. Wardinski ${ }^{1,2}$ (1) H. Amit ${ }^{2}$, B. Langlais ${ }^{2}$ (D) and E. Thébault ${ }^{3}$ (i) \\ ${ }^{1}$ Institut Terre et Environnement Strasbourg, Ecole et Observatoire des Sciences de La Terre, Université de Strasbourg, \\ CNRS, Strasbourg, France, ${ }^{2}$ Laboratoire de Planétologie et Géodynamique, Université de Nantes, Université d'Angers, \\ CNRS, Nantes, France, ${ }^{3}$ Université Clermont Auvergne, CNRS, IRD, OPGC, LMV, Clermont-Ferrand, France
}

\begin{abstract}
Previous models of Mercury's core magnetic field based on high altitude data from first MESSENGER flybys revealed an axisymmetric structure of the field. Here, we use low altitude MESSENGER data covering the entire mission period to construct spherical harmonic models based on various spatial norms. Although we find a dominantly axisymmetric field, our models nevertheless include detectable deviations from axisymmetry. These non-axisymmetric features appear at high latitudes, resembling intense geomagnetic flux patches at Earth's core-mantle boundary. Based on this core field morphology, we then attempt to infer Mercury's internal structure. More specifically, assuming that Mercury's high-latitude non-axisymmetric features are concentrated by downwellings at the edge of the planet's inner core tangent cylinder, and accounting for the presence of a stably stratified layer at the top of Mercury's core, we establish a relation between the inner core size and the thickness of the stratified layer. Considering plausible ranges, we propose that Mercury's inner core size is about $500-660 \mathrm{~km}$, which corresponds to a stratified layer thickness of 880-500 km, respectively.
\end{abstract}

Plain Language Summary Measurements of the magnetic field of Mercury taken by the MESSENGER space probe allow us to construct a model of the magnetic field generated inside Mercury. This internal field is generated within the core of Mercury by a magnetic dynamo process. This field is highly symmetric with respect to the axis of rotation, but very much weaker than Earth's magnetic field. Deviations from the axisymmetry of the field allow us to infer the internal structure of Mercury's core. A combined interpretation of Mercury's gravity field observations and our results provide a certain range for Mercury's inner core size, which is likely to be solid. We also infer the size of Mercury's dynamo and the thickness of the stratified layer above the dynamo region. We find that Mercury's inner core size is about 500-660 km, which corresponds to a stratified layer thickness of $880-500 \mathrm{~km}$, respectively. The size of the dynamo region is between 680 and $900 \mathrm{~km}$. This study provides new insights to the internal structure of a planet's core that are inferred from observations of its magnetic field.

\section{Introduction}

Based on Mariner and MESSENGER satellite missions, it was found that Mercury's internal magnetic field is very weak, dipole dominated, largely axisymmetric and with a magnetic equator shifted northward with respect to the geographic equator at midlatitudes of the northern hemisphere (Anderson et al., 2011, 2012; Johnson et al., 2012; Ness, 1979; Ness et al., 1974; Oliveira et al., 2015; Thébault et al., 2018; Wardinski et al., 2019), which is challenging to explain in terms of core structure and dynamics. In addition, several studies of Mercury's interior based on analyses of MESSENGER gravity field measurements and libration data suggested that the top of Mercury's outer core is stably stratified (Dumberry \& Rivoldini, 2015; Smith et al., 2012). Likely, this layer is comprised of $\mathrm{FeS}$, but its phase (liquid or solid) remains uncertain. Thermal stratification implies that the heat flux at the core surface is sub-adiabatic while compositional stratification implies the presence of light elements below the core-mantle boundary, both of which have important implications for the inner core solidification and the magnetic field generation.

Numerical dynamo simulations may provide further insight into Mercury's core structure. Possible scenarios include deep-seated dynamos below a thick stable layer (Christensen, 2006; Christensen \& Wicht, 2008; Takahashi et al., 2019), thin-shell dynamos (Stanley et al., 2005), and dynamos with a thin stratified layer (Stanley \& Mohammadi, 2008). The weakness of Mercury's core field motivated modeling a sulfur-rich liquid core with different zones of Fe-precipitation, that is, iron snow. These zones could exist at the bottom of the liquid core or 
at the midrange between the inner and outer core boundaries (Vilim et al., 2010). While numerical dynamos with a stably stratified layer at the top of the shell can explain Mercury's weak magnetic field and its axisymmetry, additional ingredients are needed to explain the northward shift of the magnetic equator. Numerical dynamos with imposed heterogeneous heat flux in the form of equatorial cooling at the outer core boundary (Cao et al., 2014) lead to a convective instability and an offset of the magnetic equator, but their magnetic fields are too energetic and non-axisymmetric. In contrast, Tian et al., 2015 imposed a degree-1 axially heterogeneous heat flux on a dynamo model with a stratified layer at the top of the shell and hyper-diffusivity to obtain more Mercury-like magnetic fields. In both cases the validity of the results relies on the actual pattern of thermal heterogeneity at the base of Mercury's mantle, which is largely uncertain. Double diffusive convection phenomena have also been considered to explain Mercury's magnetic field. These phenomena occur when the convection is driven by two sources of buoyancy, that is, temperature and composition (Manglik et al., 2010). Recently, Takahashi et al., 2019 showed that a double-diffusive convecting shell surrounded by a thick thermally stably stratified layer can generate Mercury-like magnetic fields. Furthermore, numerical dynamo models of Mercury's magnetic field provide estimates of the size of Mercury's inner core. Cao et al., 2014 suggested an inner core radius smaller than $1,000 \mathrm{~km}$. Based on geodetic analyses Dumberry \& Rivoldini, 2015 gave an upper limit on the inner core size of $650 \mathrm{~km}$, with the outer core dynamics partly consisting of snow formation.

These different scenarios of Mercury's dynamo lead to characteristics that should be testable by observations of space-borne magnetometers like MESSENGER and Bepi-Columbo. A careful processing and analysis of magnetic field measurements taken in planetary environments is crucial for the identification of such magnetic field characteristics.

Mainly, two techniques have been applied to study Mercury's magnetic fields: potential field methods such as spherical harmonics (Uno et al., 2009; Wardinski et al., 2019), spherical caps (Thébault et al., 2018) or equivalent source dipoles (Oliveira et al., 2015) that restrict the analysis to those observations obtained in a source free region, and (reduced) parametric models that infer the magnetic dipole moment from the space probe's magnetic equator crossing, that is, where the radial field $B_{r}$ is zero, far from the planet (Anderson et al., 2012; Johnson et al., 2012). The latter method provides models with a reduced set of parameters and is popular because of its relative independence of the data distribution. Data used for these reduced parametric models sampled the magnetic field in the magnetospheric region, with a considerable electrical current density that requires additional assumptions about the geometry and distribution of local current systems (Connerney \& Ness, 1988). In contrast, Uno et al., 2009 showed by inverting synthetic data from numerical dynamo simulations that a spherical harmonic analysis can recover the large-scale magnetic field from hemispherical uneven data distribution, as single MESSENGER flybys, when data are taken in a source-free region. The resolution of finer details of the magnetic field needs, off course, numerous orbital tracks.

In this study, magnetic field data are used to derive field models that may constrain the internal structure of a planet. The downward continuation of a magnetic field model to the core surface reveals patterns of magnetic flux. In particular, the latitude at which intense flux patches are concentrated may indicate the size of the inner core. Intense flux concentrations near the intersection of the inner core tangent cylinder are prominent in the geomagnetic field for at least the last 400 years (e.g., Jackson et al., 2000) and possibly over the past tens of millennia (see (Panovska et al., 2019) and references therein). Numerous studies explored the kinematics as well as the dynamical origin of intense high-latitude flux patches in geomagnetic field models and numerical dynamos (Amit et al., 2010, 2011; Bloxham et al., 1989; Christensen et al., 1998; Olson et al., 2018; Peña et al., 2016). The latitude at which these flux concentrations occur has been related to the change of the dynamical regime at the tangent cylinder that is coaxial with the rotation axis and tangential to the inner core boundary (Gubbins \& Bloxham, 1987), while the longitude at which these flux patches occur may be controlled by thermal core-mantle interactions (Bloxham \& Gubbins, 1987).

Here, we will use inferences from the Earth's core to carefully establish the relation between the latitude of intense magnetic flux patches and the tangent cylinder intersection with the core-mantle boundary (CMB), including possible errors associated with time-dependence and variability from one patch to another. We will then account for the existence of stratification to relate the depth of the stable layer with the radius of the inner core for a given latitude of magnetic flux patches. This relation will be implemented for the case of Mercury's magnetic field. 

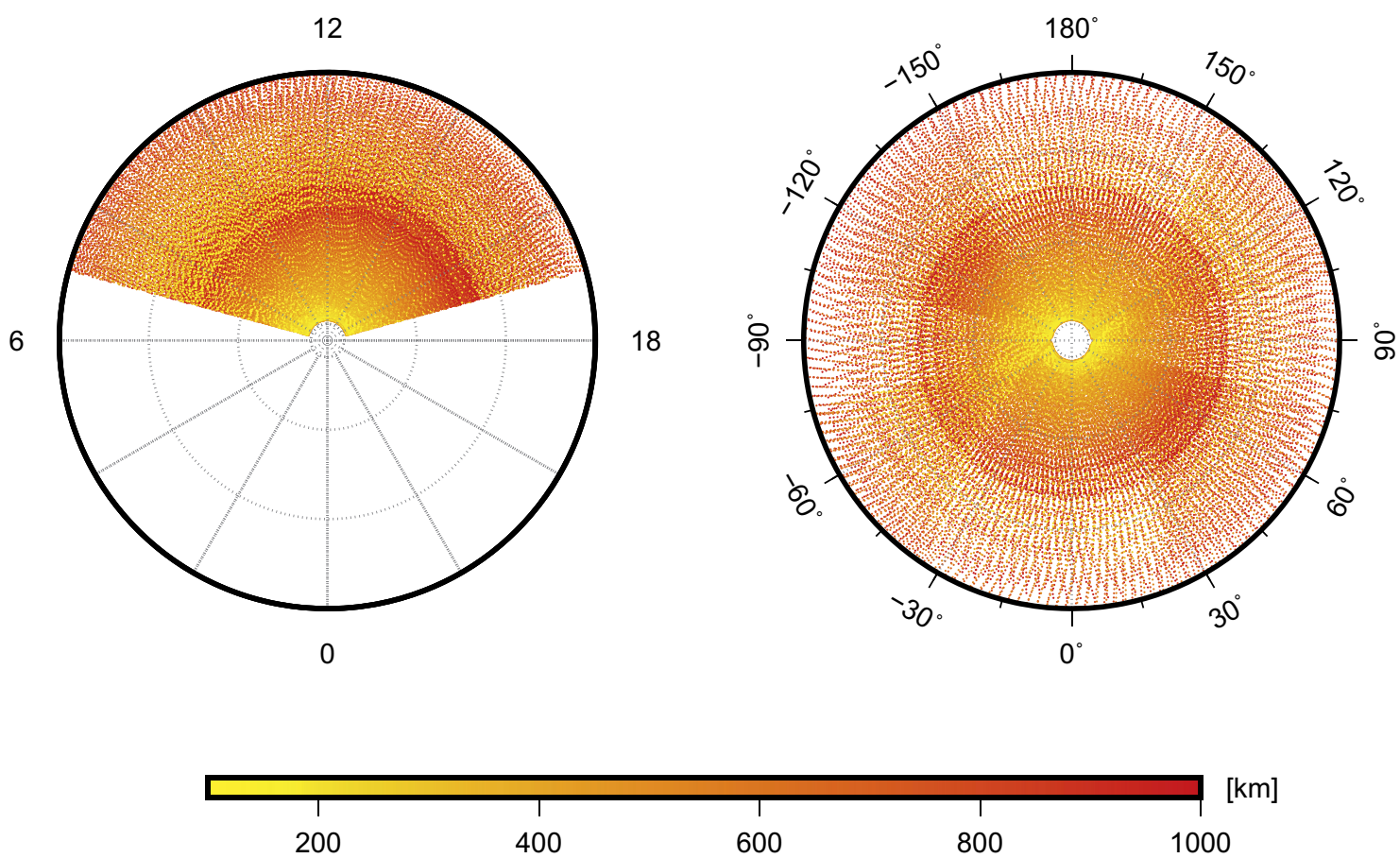

Figure 1. Spatial distribution of the selected MESSENGER data. The left panel shows the distribution over the northern hemisphere in the Mercury-Sun oriented coordinate system with respect to local time, where local time 0 is in Sun-direction. The right panel displays the data distribution over the northern hemisphere in the Mercury body-fixed coordinate system. In both panels the altitude of the data points is color-coded.

The aim of this study is twofold: First, we explore to what extent intermediate-scale spatial features of Mercury's magnetic field can be retrieved from the MESSENGER data by applying a spherical harmonic analysis; second, we aim to infer the internal structure of Mercury's core and the convective state of its dynamo. The paper is organized as follows: The description of the data and their selection is given in Section 2, Section 3 briefly describes the spherical harmonic modeling method, and results are provided in Section 4. Implications for the generation of Mercury's core field and the structure of its core are discussed in Section 5. We summarize our main findings in Section 6.

\section{Data Selection}

The MESSENGER spacecraft was in orbit around Mercury from 18 March 2011 to 30 April 2015. The orbit of MESSENGER was highly eccentric, with periapsis ranging from 200 to $500 \mathrm{~km}$ over the north polar region, and apoapsides of $>12,700 \mathrm{~km}$ above the southern hemisphere (orbital period 12hr, 4,000 orbits). This highly eccentric orbit led to an uneven data distribution, where only measurements over the northern hemisphere are assumed to be inside the magnetospheric cavity which allow adequate modeling of Mercury's internal magnetic field (Oliveira et al., 2015). All local times are covered within 88 (terrestrial) days.

Here, we selected data from a satellite altitude range of 100-1,000 km during local night-time from 7:00 p.m. to 5:00 a.m. This provides 13,60,051 vector triplets, which consist of measurements of Mercury's magnetic field in three directions $B_{\theta}, B_{\phi}$ and $B_{r}$ (see Appendix A for stricter selection schemes). Figure 1 shows the spatial distribution of the selected data set in two coordinate systems and the distribution with respect to local time. The altitude of the data (color-coded) is in the range from 100 to $1,000 \mathrm{~km}$. There is a polar gap with a diameter of $\sim 3$ degrees around the North Pole, which is not covered by satellite measurements.

The nighttime selection criterion is often used in the derivation of geomagnetic field models that are based on satellite data (Finlay et al., 2016; Lesur et al., 2008; Olsen et al., 2006). This has proven to provide data sets with largely removed external field contamination and to allow a precise description of Earth's core field to high spherical harmonic degrees. To this aim, we apply the same selection criterion to the MESSENGER data set. The altitude selection criterion guarantees that the analyzed magnetic field measurements are within the 
magnetospheric cavity: $1,000 \mathrm{~km}$ is smaller than the averaged subsolar distance of the magnetopause location (Thébault et al., 2018; Winslow et al., 2013). Oliveira et al., 2019 showed that the crustal magnetic signal is small-scale and weak in amplitude at $40 \mathrm{~km}$ altitude. Therefore, at $100 \mathrm{~km}$ altitude signals due to crustal magnetization are assumed to be negligible at large length scales. The combination of both criteria provides a data set, which shows negligible crustal field signatures and the least contamination from magnetospheric and exospheric magnetic fields (Wardinski et al., 2019), which are strong at the planet's day-side. We discuss further data selection schemes and their impact onto the magnetic field models in Section 4.3 and Appendix A.

\section{Method}

All of the selected data over the northern hemisphere (see Section 2) sampled Mercury's magnetic field in a region with almost no magnetic sources. Therefore, a potential theory and spherical harmonic analysis provides adequately a separation between external and internal magnetic field sources. We seek to fit MESSENGER observations of Mercury's magnetic field by a potential that is parameterized using spherical harmonics, that is,

$$
\begin{aligned}
V= & a \sum_{l=1}^{L_{\mathrm{int}}} \sum_{m=0}^{l}\left\{\left(g_{l}^{m} \cos (m \phi)+h_{l}^{m} \sin (m \phi)\right)\left(\frac{a}{r}\right)^{l+1} P_{l}^{m}((\cos \theta))\right\} \\
& +a \sum_{l=1}^{L_{\mathrm{ext}}} \sum_{m=0}^{l}\left\{\left(q_{l}^{m} \cos (m \phi)+s_{l}^{m} \sin (m \phi)\right)\left(\frac{r}{a}\right)^{l} P_{l}^{m}(\cos \theta)\right\}
\end{aligned}
$$

where $a$ is Mercury's radius (2,440 km). $r$ is the radial distance from Mercury's center, $\theta$ the colatitude, and $\phi$ the longitude. $P_{l}^{m}(\cos \theta)$ are the Schmidt semi-normalized associated Legendre functions, where $l$ is the degree and $m$ the order. $L_{\mathrm{int}}$ and $L_{\mathrm{ext}}$ are the truncation degrees of the spherical harmonic expansions for the internal and external field, respectively. The Gauss coefficients $\left\{g_{l}^{m}, h_{l}^{m}\right\}$ and $\left\{q_{l}^{m}, s_{l}^{m}\right\}$ represent the internal and external magnetic field, respectively. These model parameters are estimated by a least squares fit to data collected during a given time interval. In the following, we outline details of our modeling technique, which is sometimes called smoothed inversion (Holme \& Bloxham, 1996; Uno et al., 2009). From the selected data, we derive models with $L_{\text {int }}=10$ and $L_{\text {ext }}=1$. External magnetic fields of higher spherical harmonic degrees cannot be estimated with confidence by using a regularized inversion. Their signals should contribute to the model residuals, that is, un-modeled signals. These un-modeled signals could be partly related to the magnetic signatures of Birkeland currents, which mainly exist in the dawn and dusk sections of the data local times at latitudes higher than $70^{\circ}$ north. Their signals are generally in the horizontal field components, with magnitudes of only $20 \mathrm{nT}$ and they do not rotate with the planet (Anderson et al., 2018).

\subsection{Model Priors}

For a linear least squares problem the model vector $m$ containing the Gauss coefficients is found at the minimum of an objective function

$$
\Theta(m)=(\mathbf{y}-\mathbf{A} m)^{\top} \mathbf{C}_{\mathbf{e}}^{-1}(\mathbf{y}-\mathbf{A m})+\lambda_{S}\left(\mathrm{~m}^{\top} \mathbf{C}_{\mathbf{m}}^{-1} \mathrm{~m}\right)
$$

where $y$ is the data vector, $\mathbf{A}$ a design matrix, $\mathbf{C}_{\mathbf{e}}$ the data error covariance matrix, and $\mathbf{C}_{\mathbf{m}}$ the prior model covariance matrix (Gubbins, 1983; Jackson, 1979), which is controlled by a Lagrange multiplier $\left(\lambda_{S}\right)$. The misfit of the model is computed by

$$
\hat{\sigma}=\sqrt{\frac{\sum_{i=1}^{N}\left(y_{i}-\hat{y}_{i}\right)^{2}}{N-1}},
$$

where $\hat{y}_{i}$ is the model value for given observation $y_{i}$.

Our method to find the Gauss coefficients utilizes prior constraints to reduce the ambiguity of the data inversion. The application of priors to constrain the inversion of MESSENGER data to obtain a model of Mercury's magnetic field is justified by their uneven hemispherical distribution. Primarily, the prior should emphasize the largescales of Mercury's magnetic field. We test the performance of four different priors of the spatial complexity of the model's field morphology. These priors are usually formulated as model norms: 


$$
\begin{aligned}
& \text { Norm 1: }\left.\oint B^{2} d S\right|_{r=c}=(l+1)\left(\frac{a}{c}\right)^{(2 l+4)} \\
& \text { Norm 2: }\left.\oint B_{r}^{2} d S\right|_{r=c}=\frac{(l+1)^{2}}{2 l+1}\left(\frac{a}{c}\right)^{(2 l+4)} \\
& \text { Norm 3: }\left.\oint\left(\nabla_{h} B_{r}\right)^{2} d S\right|_{r=c}=\frac{l(l+1)^{3}}{2 l+1}\left(\frac{a}{c}\right)^{(2 l+6)} \\
& \text { Norm 4 }\left.\oint F d S\right|_{r=c}=\frac{(l+1)(2 l+1)(2 l+3)}{l}\left(\frac{a}{c}\right)^{(2 l+3)} \leq Q .
\end{aligned}
$$

In Norm 4 of Equation $4 F$ is the field intensity and $Q$ is the mean CMB heat flux. In all these expressions in Equation 4, Mercury's core radius is $c=2,060 \mathrm{~km}$ (Wardinski et al., 2019). Norm 1 minimizes the power of the magnetic field for higher spherical harmonic degrees and therefore it steepens the slope of its power spectrum. Norms 2 and 3 smooth the radial magnetic field and its horizontal gradient, respectively (Shure et al., 1982). Norm 4 is different from the other norms as it may include prior knowledge of the heat flux at Mercury's core surface, which is due to the Ohmic dissipation of the radial field $B_{r}$ at the core surface (Gubbins, 1975). However, there are no observations of the heat flux at Mercury currently available. Therefore, Norm 4 acts merely as a constraint to stabilize the solution of the inversion like the other norms. All norms dim the amplitude of small-scale field features, though at different ways, hence support the large-scale morphology of the magnetic field. Among these norms, this effect is most strongly imposed by Norm 3, where the attenuation scales with $1^{3}$.

The resulting model is determined by varying the strength of the prior to be in optimal balance between data misfit and model smoothness. This optimal balance is usually found for the $\lambda_{S}$ at the knee of their trade-off or L-curves.

\subsection{Iterative Modeling Scheme}

To find the model parameters, we adopt an iterative reweighting scheme that consists of three steps. At the first step, we determine a model that is based on data covering the MESSENGER's entire mission interval at Mercury. Data are weighted equally, to form the initial error covariance matrix, $\mathbf{C}_{\mathbf{e}}$, in Equation 2. We assign an initial error of $1.6 \mathrm{nT}$ to each datum, which corresponds to the upper limit of the instrument's resolution (Anderson et al., 2007). At a second step, individual differences between each data and corresponding values of the initial model are computed, to provide an update of $\mathbf{C}_{\mathbf{e}}$ and, third, to derive the final model with the updated error covariance matrix. The residual amplitude, and therefore $\mathbf{C}_{\mathbf{e}}$, depends directly on the Lagrange multiplier $\lambda_{S}$; In order to obtain a close trade-off curve for each norm, this iterative reweighting scheme is applied for each setting of $\lambda_{S}$. In total we derive a large number of models for each norm. We select the model at the knee of each norm trade-off curve.

A closer inspection of the residuals reveals anomalous tracks that show significant larger residual amplitudes than others. The cause for these large residuals remains unclear, but could be related to instrument errors and/or data processing errors. However, these data are automatically down-weighted. About 6000 data points with large misfits, that is, $>200 \mathrm{nT}$ are rejected.

\subsection{Robustness of the Solutions}

There are a few diagnostics to evaluate the robustness and confidence of the inversion results. First, we analyze the resolution matrix of the model $\mathrm{m}$, to obtain a measure of model parameter significance. The resolution matrix is given by

$$
\mathbf{R}=\left(\mathbf{A}^{\top} \mathbf{C}_{\mathbf{e}}^{-1} \mathbf{A}+\lambda_{s} \mathbf{C}_{\mathbf{m}}^{-1}\right)^{-1} \mathbf{A}^{\top} \mathbf{C}_{\mathbf{e}}^{-1} \mathbf{A},
$$

where the diagonal elements of $\mathbf{C}_{\mathbf{m}}$ are defined in Equation 4. Ideally, this matrix would be an identity matrix. A regularization scheme must be applied to solve the ill-posed inverse problem and to obtain a stable solution. This is reflected in the form of the resolution matrix. A value of the resolution near one means that a model parameter is wholly determined by the data. Decreasing values correspond to increasing control of the prior information. 


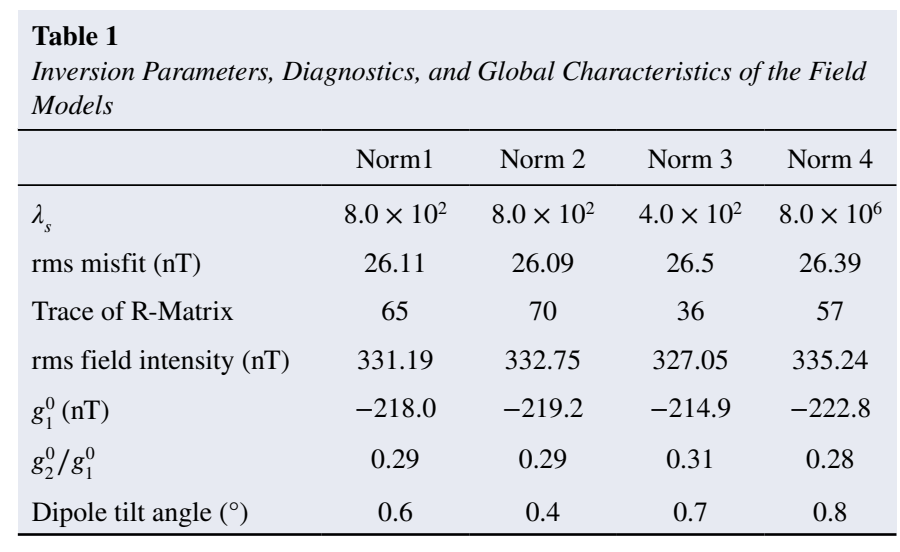

The trace of the resolution matrix $\operatorname{Tr}(\mathbf{R})$ can be broadly interpreted as the degree of freedom of the model and as the number of model parameters resolved by the inversion (Tarantola, 1987).

Characteristics and diagnostics of the field models are listed in Table 1. All models widely agree in their statistical properties and mostly differ in their numbers of resolved parameters. The lowest number of resolved parameters is found for Norm 3, as it more strongly damps contributions of higher spherical harmonic degrees than other norms and therefore reduces the degree of freedom most strongly. See Appendix B for a further discussion of the inversion covariance matrix.

\section{Results}

In this section, we present models of Mercury's steady magnetic field as they are based on MESSENGER measurements covering the period 2011-2015. We emphasize that this steady field model that was obtained from data covering a short period of time does not mean that Mercury's field is static. We discuss to what extent our results are conclusive and estimate their robustness. The models provide reasonably good fits to the data with residuals of $\sim 8 \%$ of the total field strength.

\subsection{Resolution Analysis and Spectral Content}

For each of the four resulting models we computed the resolution matrix $\mathbf{R}$ and charted their diagonal elements, where labels of the models refer to the norm used to constrain the solution, for example, Norm $1 \rightarrow$ Model 1 . These plots (resolution curves) are shown in Figure 2.

The resolution curves of all models generally agree for the first four spherical harmonic degrees. Most notable are the high resolution of sectorial Gauss coefficients, that is, $g_{m}^{m}, h_{m}^{m}$, of at least the first five spherical harmonic degrees. Resolution curves of models 1, 2, and 4 show this particular pattern also for higher spherical harmonic degrees, which may indicate a higher ability of these norms to capture small-scale magnetic field signatures. The higher resolution of the sectorial terms could also be explained by MESSENGER's flight path along latitude. We find that Model 3 resolves magnetic field structures only until spherical harmonic degree 5. Model 4 partly resolves spherical harmonic degree 7, whereas Models 1 and 2 show a higher resolution and partly resolve degree 8 . Vertical lines in Figure 2 mark the maximum number of resolved model parameters corresponding to the trace of the resolution matrix (see Table 1).

This coincides with a resolution level of $\sim 0.4$. Below this level, the model is assumed to be dominated by the prior. At degree 10, that is, coefficient numbers between 100 and 120, Models 1, 2, and 4 show an enhanced resolution, where they may become sensitive to spectral leakage, signals of other sources, and possible data errors. We assume the high resolution at these small scales to be an artifact and possibly caused by the orbital geometry of MESSENGER. Holme and Bloxham 1996 discussed a similar effect observed in the Voyager II data at Neptune, which was likely caused by the spacecraft trajectory. The setting of a maximum degree $L_{\text {int }}$ for the spherical harmonic expansion leads also to an aliasing of the higher degree field $\left(l>L_{\text {int }}\right)$ into coefficients of the model spherical harmonic expansion (spectral leakage). We assume this effect to be reduced by truncating the models to spherical harmonic degree $L_{\text {int }}=8$.

Maps derived from truncated models $(l=8)$ of the radial magnetic field at the core surface are shown in Figure 3. As expected, field structures in the northern hemisphere show more details than in the southern hemisphere, because of the data distribution. Generally, the field is dominated by the axial dipole and the magnetic equator is significantly shifted towards the North Pole in agreement with previous studies (Anderson et al., 2011, 2012; Thébault et al., 2018). However, the models show differing spatial complexities, in particular of the magnetic equator. The map derived with Norm 3 shows the
Figure 2. Diagonal elements of the resolution matrix of the preferred models for different priors versus coefficient number (see spherical harmonic degrees at the top axis). Gray shaded areas indicate even spherical harmonic degrees. The colored vertical lines represent the degree of freedom of the respective model and the black solid horizontal line marks a resolution of 0.4 . 
(a)

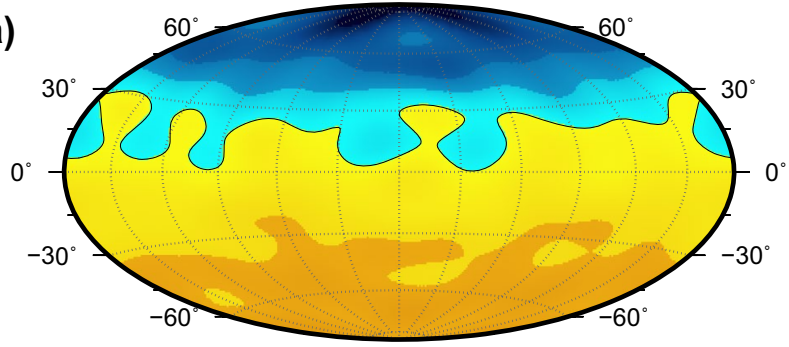

(c)

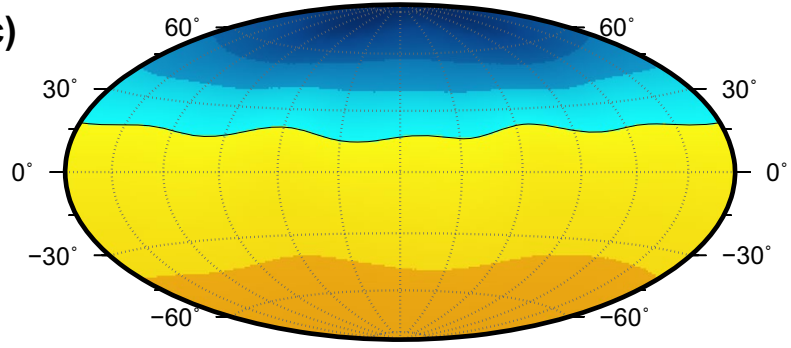

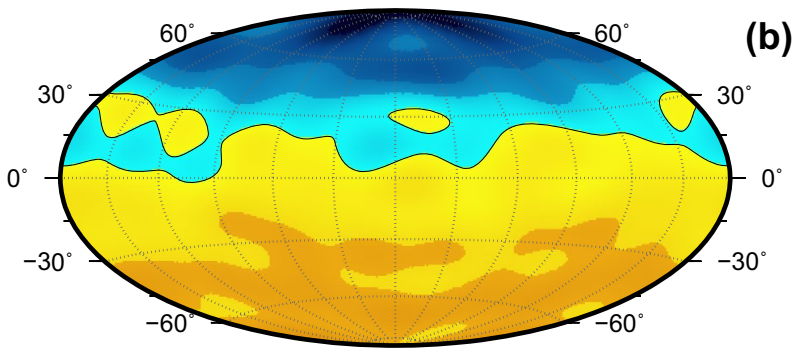

(b)

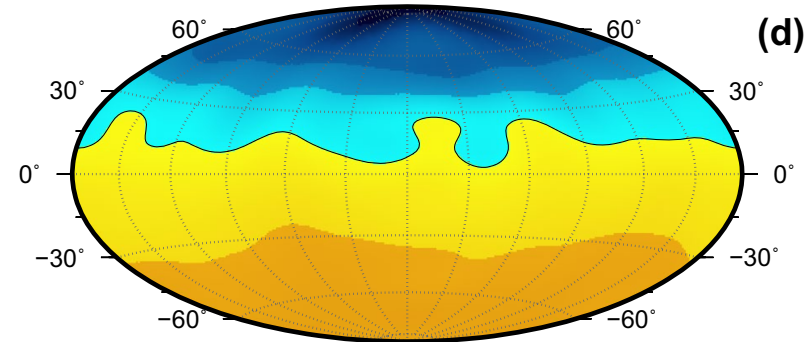

(d)

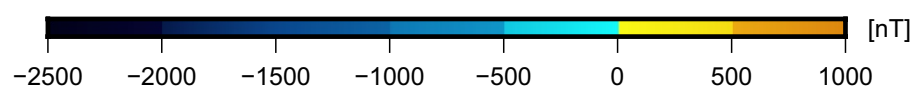

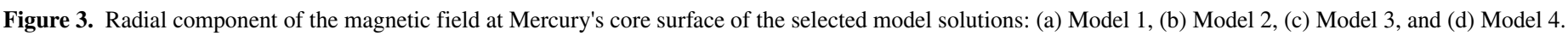

most axisymmetric field morphology, whereas models derived with Norms 1 and 2 show more longitude-dependent structures including even some reversed flux patches. Overall, all models tend to agree in their large-scale structure (Table 1) and differ in their quantification of small-scale features.

All models (1-4) show axial quadrupole-dipole ratios of 0.28-0.31 (Table 1), which are significantly smaller than those obtained by dipole offset models (Anderson et al., 2012; Johnson et al., 2012) but are in agreement with a model constructed using spherical caps (Thébault et al., 2018). We note that we could force the models to have a larger quadrupole-dipole ratio close to $g_{2}^{0} / g_{1}^{0}=0.4$ (found by Anderson et al., 2012; Johnson et al., 2012); however, this leads to $10 \%-20 \%$ larger rms misfits, which we consider to be significant and eventually deleterious.

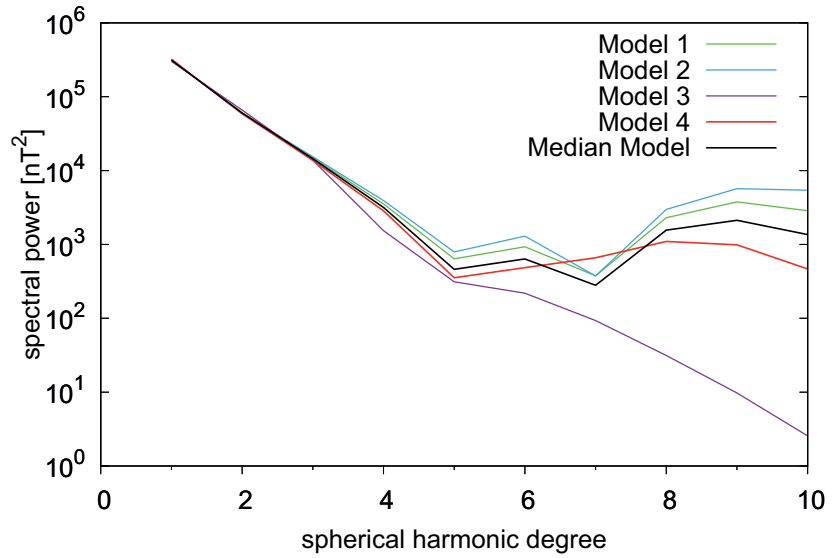

Figure 4. Power spectra of the magnetic field models at Mercury's core surface.
Maps in Figure 3 also have structures where no data are available, that is, in the southern hemisphere. The magnetic field morphology in this hemisphere is mostly determined by the global characteristic of the spherical harmonic analysis and the prior used in the inversion. We therefore do not attempt to interpret magnetic field features in the southern hemisphere.

Power spectra (Lowes, 1966; Mauersberger, 1956) of the models at the core surface are shown in Figure 4. The spectra mostly match for spherical harmonic degrees 1 to 3 . We find three different types of spectral slopes for spherical harmonic degrees $l>5$ : decreasing, increasing, and flat. The spectral power of Model 3 drops exponentially, whereas Models 1 and 2 show powers that increase by one order of magnitude. Model 4 shows a flatter spectrum. This may lead to similar conclusions as taken from the resolution analysis, where for Models 1, 2, and 4 spherical harmonic degrees above $l>8$ may be influenced by spectral leakage of magnetic small-scale sources close to the surface of Mercury.

Common characteristics of all models are best represented by their median of the models. This technique is commonly used in the derivation of the 
International Geomagnetic Reference Field model (IGRF), where a wide variety of geomagnetic main field models based on diverse modeling philosophies are averaged (Thébault, Finlay, Alken, et al., 2015). The discussion of the spatial characteristic of Mercury's steady magnetic field will therefore be based on this median model, which should neither be too damped (as Model 3) nor too contaminated by spectral leakage at high spherical harmonic degrees (as probably Models 1 and 2). As mentioned earlier (see discussion of Figure 2), we are not convinced by the robustness of spherical harmonic degrees $9 \& 10$, and therefore we truncate the median model to spherical harmonic degree 8.

\subsection{Mercury's Steady Magnetic Field}

The individual models are plotted in Figure 5. The radial component of the non-dipole magnetic field in the northern hemisphere is very similar for all maps (left column). Similarly, maps of the radial component of the non-zonal field largely agree in their morphology. Only the amplitude of the field features for Model 3 tend to be smaller than for the other model solutions. We conclude that all features used to infer the internal structure of Mercury are equally present in all model solutions and subject to the priors employed in the inversion, similar to (Holme \& Bloxham, 1996).

Maps of the median magnetic field model are presented in Figure 6, which is obtained from the spatial averaging of the four Models 1-4 until spherical harmonic degree 8. We refer to it as the steady field as it represents the averaged or invariant magnetic field over MESSENGER period of data, but it might not show detectable time variability during a short period of $\sim 5$ years. Figures $6 \mathrm{a}$ and $6 \mathrm{~b}$ show the non-dipole field and the non-axisymmetric, that is, non-zonal, field at Mercury's core surface, respectively. The mapping of the non-dipole field excludes the dipole coefficients $g_{1}^{0}, g_{1}^{1}$ and $h_{1}^{1}$, whereas the non-axisymmetric field excludes all zonal terms, that is, $g_{l}^{0}$. The non-dipole field (Figure 6a) is dominated by the equatorial-symmetric $g_{2}^{0}$ term. Though the estimate of $g_{2}^{0}$ is strongly influenced by the uneven distribution of MESSENGER data, it is the second strongest coefficient of the field. In addition, the field features at the northern hemisphere are stronger due to significant equatorial-antisymmetric terms, that is, $g_{3}^{0}$. Finally, the signature of two normal polarity flux patches at northern high latitudes is evident in Figure 6b. Recall that Figure 6b shows the non-zonal field; the two negative structures correspond to two normal polarity flux patches, while the two positive non-zonal structures are the lows in between (see Figure 6a).

The polar view map of the non-dipole radial field at the surface of Mercury's core (Figure 6c) shows an elongated patch of intense magnetic flux over the North Pole. This pattern is surrounded by a region of positive (i.e., opposite polarity) magnetic flux with some intensified patterns, where the boundary between these regions of opposite polarity, that is, the magnetic equator (black line) shows considerable undulations. These undulations further indicate non-axisymmetric field contributions.

The non-zonal field, shown in Figures $6 \mathrm{~b}$ and $6 \mathrm{~d}$, is fainter. Its amplitude ranges between $\pm 200 \mathrm{nT}$. This part of the field allows to unravel longitude-dependent structures that are otherwise masked by the strong axisymmetric field. Particularly, the non-zonal field shows four features with alternating signs at high latitudes (A, B, C, and $\mathrm{D}$ in Figure 7), indicative of two intense normal flux patches. The centers of these flux patches appear approximately at $65^{\circ}$ northern latitude.

Furthermore, Mercury's core field shows non-zonal structures at lower latitudes. These primed features (A', B', C' \& D' in Figure 7) are weaker than their higher latitude counterparts. The primed features seem to be shifted relatively to the higher latitude structures by a longitudinal angle of $30^{\circ}-60^{\circ}$ to the west. The very existence of the primed features may provide further interpretations of processes and structures within Mercury's core. However, their relative weakness might render these interpretations as too speculative.

\subsection{Model Dependence on Data Selection and Model Residuals}

Before we attempt to interpret magnetic field structures in terms of internal properties of Mercury, we discuss the possibility that other sources than internal contribute to the derived model. In Appendix A, we explore the influence of the data selection on the derived internal magnetic field model. Two schemes are set up to explicitly assess the impact of field aligned currents on the internal magnetic field configuration close to Mercury's North Pole. The most important source of an external field at the planet's night-side is related to Birkeland currents, which flow radially in the north polar region. Their magnetic signature exists only in the horizontal components 

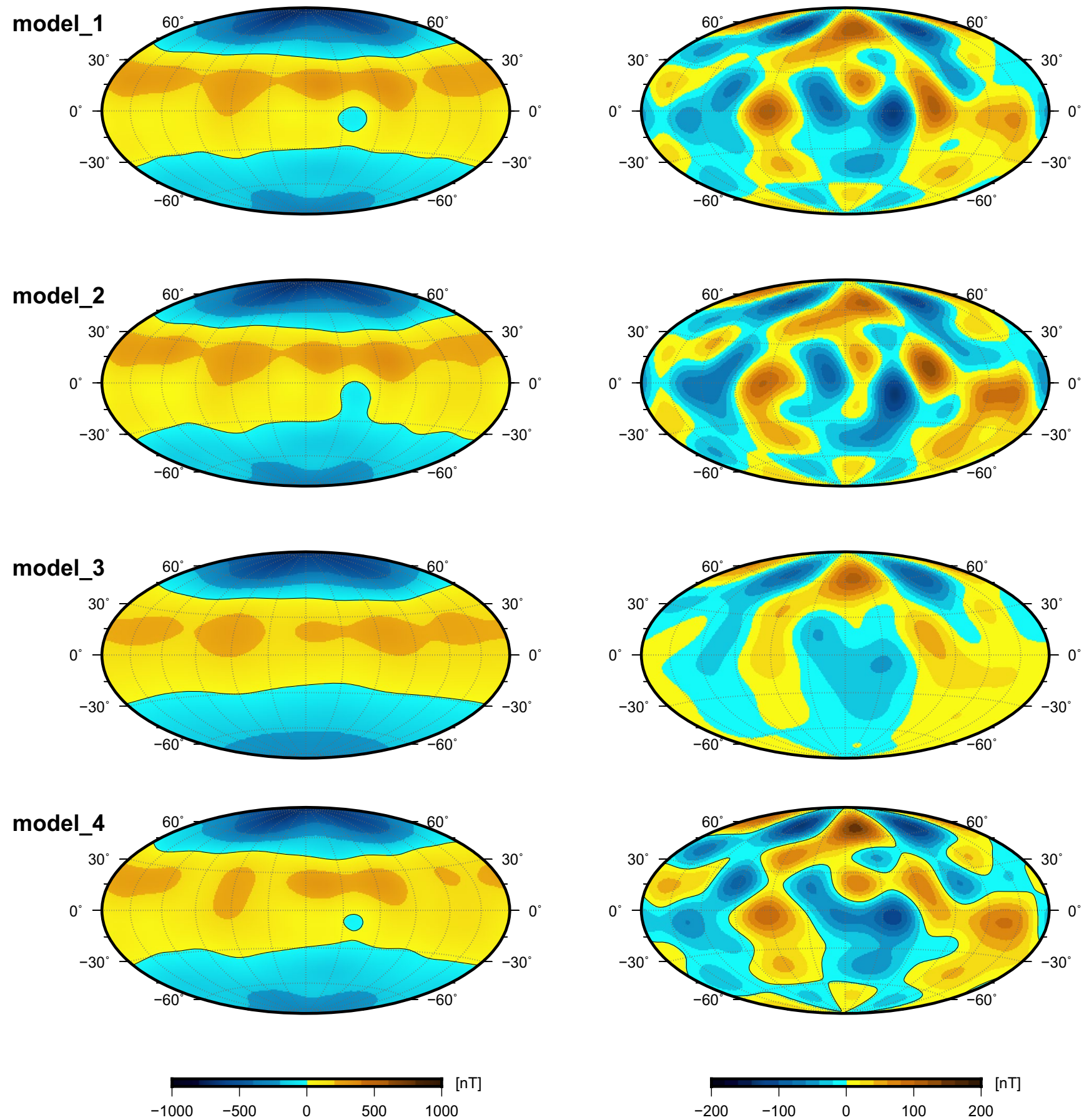

Figure 5. Maps of the non-dipole radial (left) and the non-zonal radial magnetic field (right) at Mercury's core surface for the 4 model solutions.

and would show up as an auroral (zonal) band in a time-averaged analysis. The observed amplitudes are in the range of $\pm 40 \mathrm{nT}$ (Anderson et al., 2014). Such zonal features are absent in our maps and have to be absent in $B_{r}$; instead we observe distinctive non-zonal features of Mercury's radial magnetic field. Therefore, a relation between the primed and un-primed features in Figure 7 and Birkeland currents can be ruled out.

Furthermore, we applied different data selection schemes to evaluate the dependence of the modeling results on the disturbance caused by magnetic activity in the magnetosphere of Mercury (see Appendix A). These results also suggest that features of our internal magnetic field model are robust and do not vary significantly with magnetic activity conditions. This is concluded from Figures A4-A6, where the morphology of the non-zonal radial 
(a)

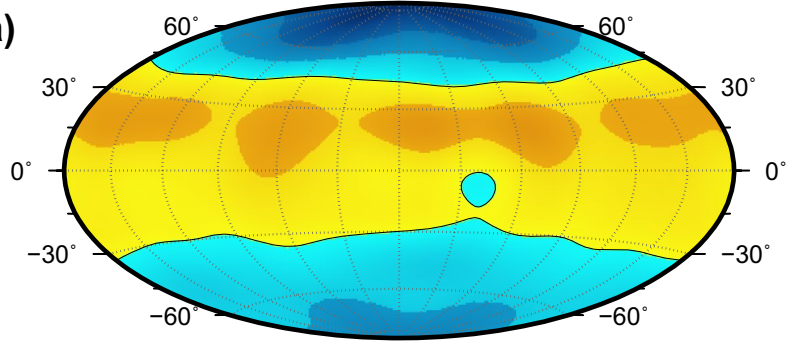

(c)

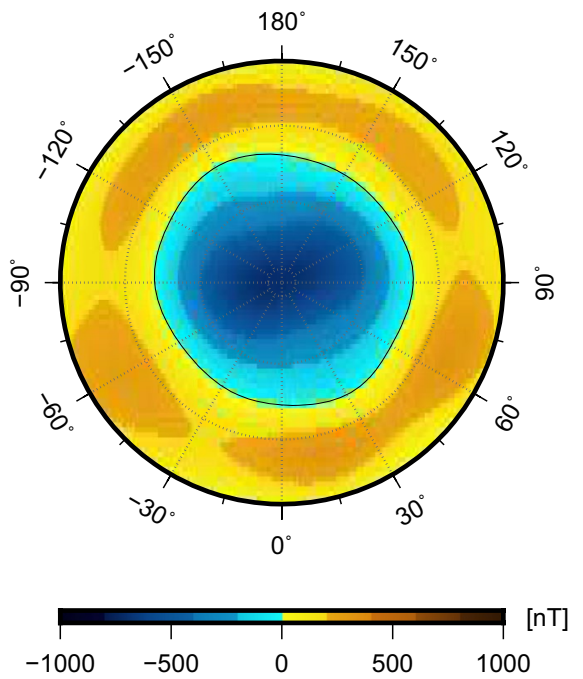

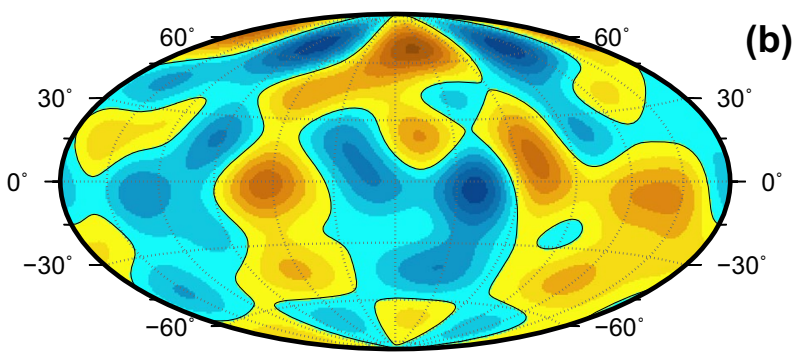

(b) $0^{\circ}$

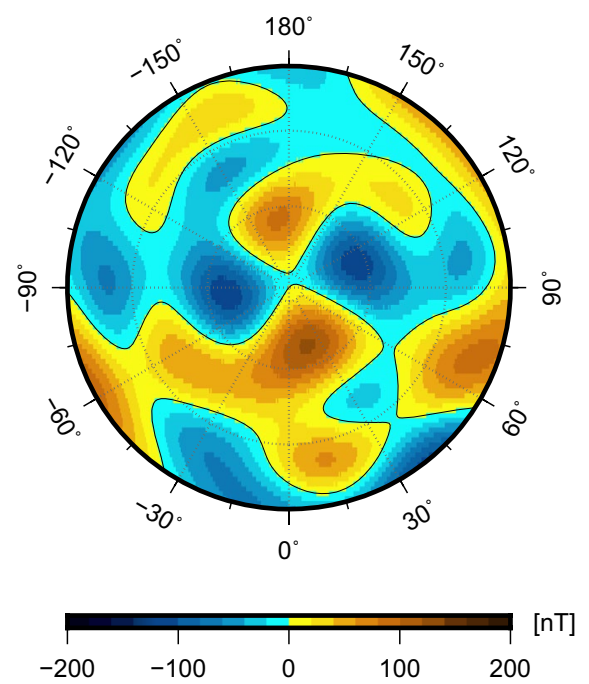

(d)

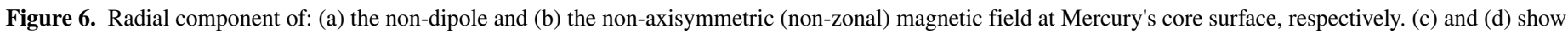

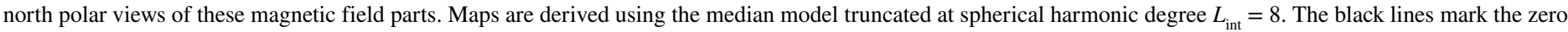
contour. Note different scales for maps (a), (c) and (b), (d).

magnetic field does not vary significantly between moderately disturbed and quiet conditions. For very quiet conditions, patches A, B, C, and D are still clearly visible in maps of Figure A6, but the field morphology slightly differs. These slight differences can be explained by a poorer spatial resolution of the optimal model for MA $<10$ due to a significant reduction of data points for very quiet magnetospheric conditions. Moreover, we note that an axial quadrupole-dipole ratio of $\sim 0.3$ is favored by data with very low magnetospheric field disturbances (see Table A1 for solutions with MA < 10).

We also compute residuals between Mercury's steady magnetic field model and the data set described in Section 2. Overall, residuals range between $\pm 25 \mathrm{nT}$ (this is about the model rms misfit), but occasionally show larger ranges. In the derivation of the model we discard data with residual amplitudes larger than \pm 200 nT. Model residuals are presented in two ways, as maps showing their distribution in the northern hemisphere in Figures 8 and 9 and as latitude-altitude plots in Figure 10. These maps and plots provide complementary information of the residual distribution and allow to characterize sources of the residual field. Residuals in the MSO coordinate (Figure 8) system are almost equally organized for different sidereal days of Mercury. This indicates that these residuals are of external origin and do not rotate with Mercury.

The largest residual amplitudes are found for the $\mathrm{B}_{\theta}$-component of the magnetic field, which tend to occur in a region from the equator to 30 degrees north, see left columns of Figures 8-10. Most interestingly, residuals of the $\mathrm{B}_{\theta}$-component intensify towards the equator and with altitude, which is a further indication that residual signals originate externally from Mercury. Their sources are above $1000 \mathrm{~km}$ from planet's surface hence of magnetospheric origin. Perhaps, these signals are caused by a current system that is flowing between dawn and dusk sections of Mercury's magnetosphere, like the cross-tail current, that was reported to flow at a radial distance of $\sim 1.22$ Mercury radius, that is, $\sim 2,500 \mathrm{~km}$ (Poh et al., 2017). The $\mathrm{B}_{\phi}$-component shows intensified residuals north of $85^{\circ}$ latitude, which are possibly related to field aligned currents and processes in the cusp region. The residual 

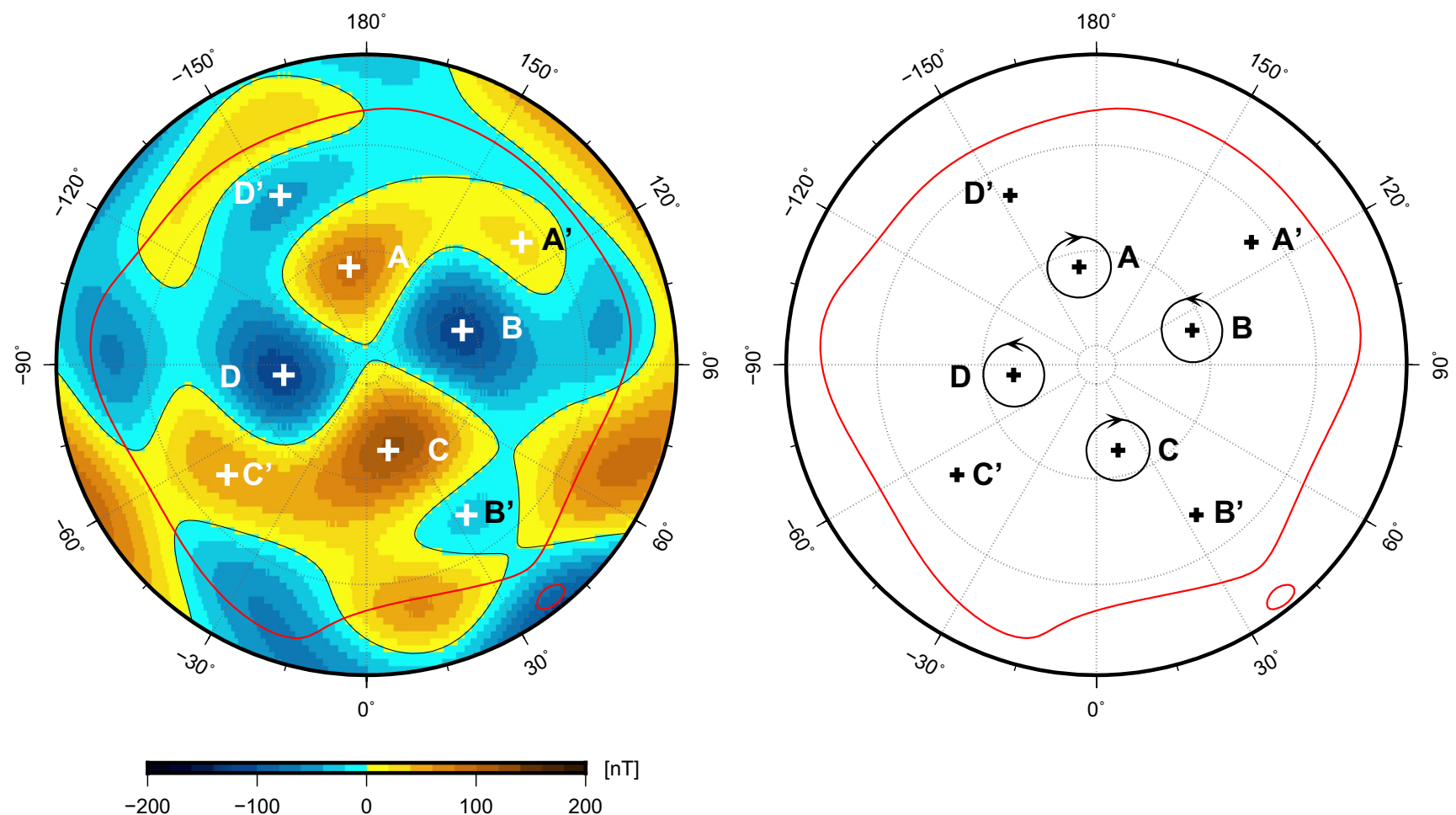

Figure 7. Polar view of the non-axisymmetric radial magnetic field at Mercury's core surface. The red line shows the position of the magnetic equator, capital letters mark apparent non-zonal field features, and pluses their centers. Right: a schematic illustration of the individual convective rolls associated with the high-latitude nonzonal field features (clockwise A, C; counterclockwise B, D), and their possibly related companion patches at lower latitudes, that is, A', B', C', and D', respectively.

amplitude of the $\mathrm{B}_{r}$-component ranges between $\pm 25 \mathrm{nT}$ and shows no distinct patterns, particularly nothing close to the non-zonal features seen in Figures $6 \mathrm{~d}$ and 7 at $\sim 65^{\circ}$ latitude.

In an altitude range from 500 to $1,000 \mathrm{~km}$ residual amplitudes and small-scale internal magnetic field features (non-zonal radial magnetic field patterns) have about the same scale. This may explain the rather small improvement in terms of rms misfit when models of Wardinski et al., 2019 are compared to those of this study (Table 1), because the rms misfit is derived at the actual altitude of the datum. The absence of known significant ionic and electric charges in this altitude range at night-side permits the application of the potential theory (e.g., Backus et al., 1996) in a source-free region. Together with the radial equally dense distributed data from 100 to $1,000 \mathrm{~km}$ altitude, this allows a robust separation of external and internal sources. Remaining signals (residuals) do not enter the model parameters and therefore are not described by the model. Different and stricter data selection schemes, which explicitly avoid magnetic signatures due to field aligned currents in the cusp region and due to magnetospheric disturbances (see Appendix A) do not alter results and support our findings of non-zonal magnetic field features and their internal origin.

\subsection{Inferring Mercury's Internal Structure}

Although our model represents a likely configuration of Mercury's internal magnetic field, its derivation has to depend on the uneven data distribution and model priors, which has to be kept in mind for the following inferences.

The concentric arrangement of non-zonal features (A, B, C, \& D) in the northern hemisphere as seen in Figure 7 could be indicative of processes that are involved in the magnetic field generation within Mercury. To reach conclusions about these processes, we assume that the nature of these features is linked to columnar rolls tangent to Mercury's inner core, parallel to the planet's spin axis that is, these columnar rolls are expected to be equatorially symmetric. Busse, 1975 showed that such columnar flow exists when the Coriolis force dominates viscous and Lorentz forces in the convective region. Oppositely rotating convective rolls (clockwise and anticlockwise, see 

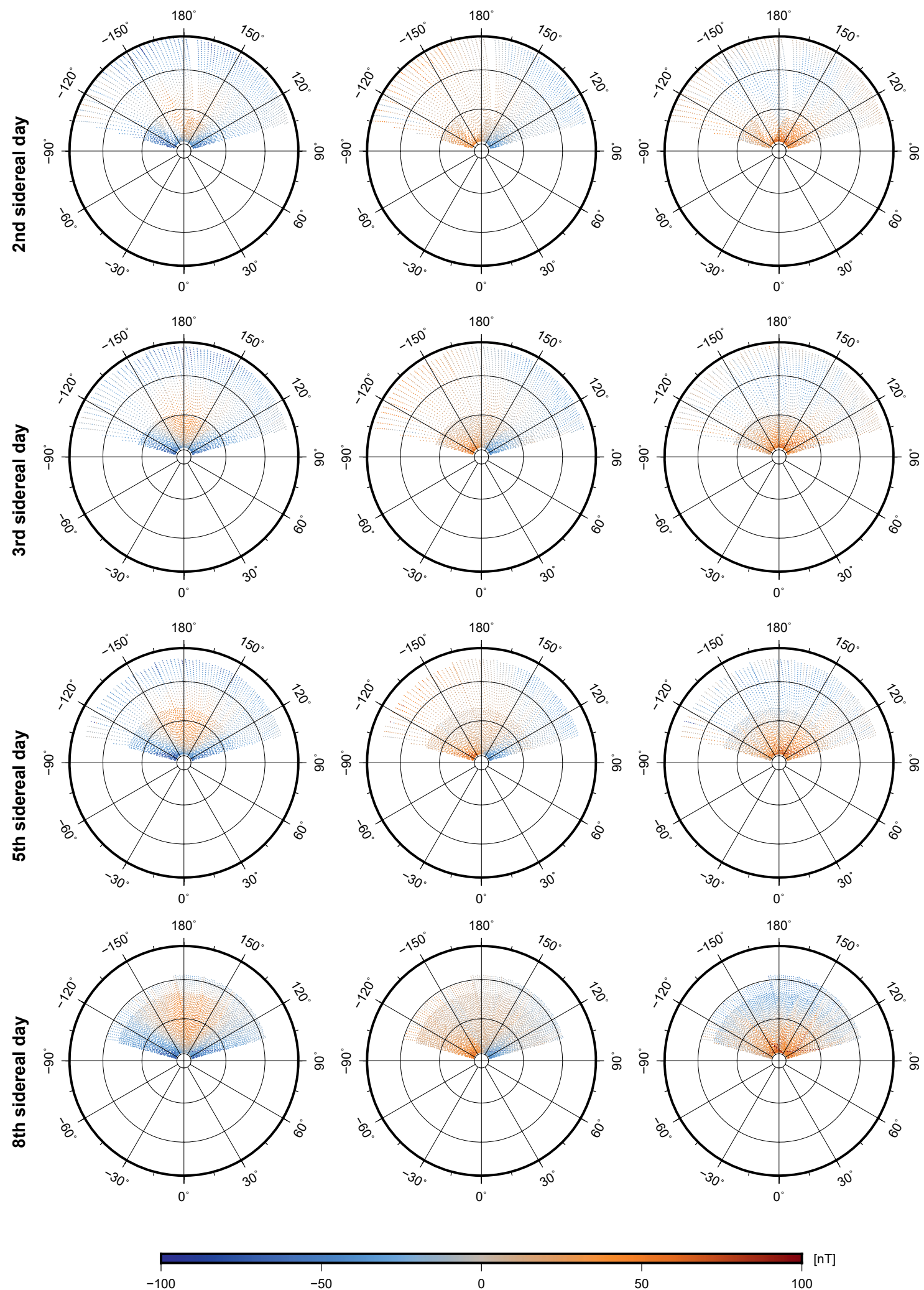

Figure 8. Maps of the model residuals at the spacecraft altitude over Mercury's northern hemisphere in a coordinate system with fixed sun orientation, the MSO coordinate system. $0^{\circ}$ longitude points towards the sun and represents noon local time, where longitudes $\pm 90^{\circ}$ represent dawn and dusk, respectively. Residuals are temporally segmented in 88-days bits, which correspond to the orbital period of Mercury around the Sun, that is, one sidereal day of Mercury. From left to right are shown the components $\mathrm{B}_{\theta}, \mathrm{B}_{\phi}$ and $\mathrm{B}_{r}$ located on the night side during 7p.m. and 5a.m. 

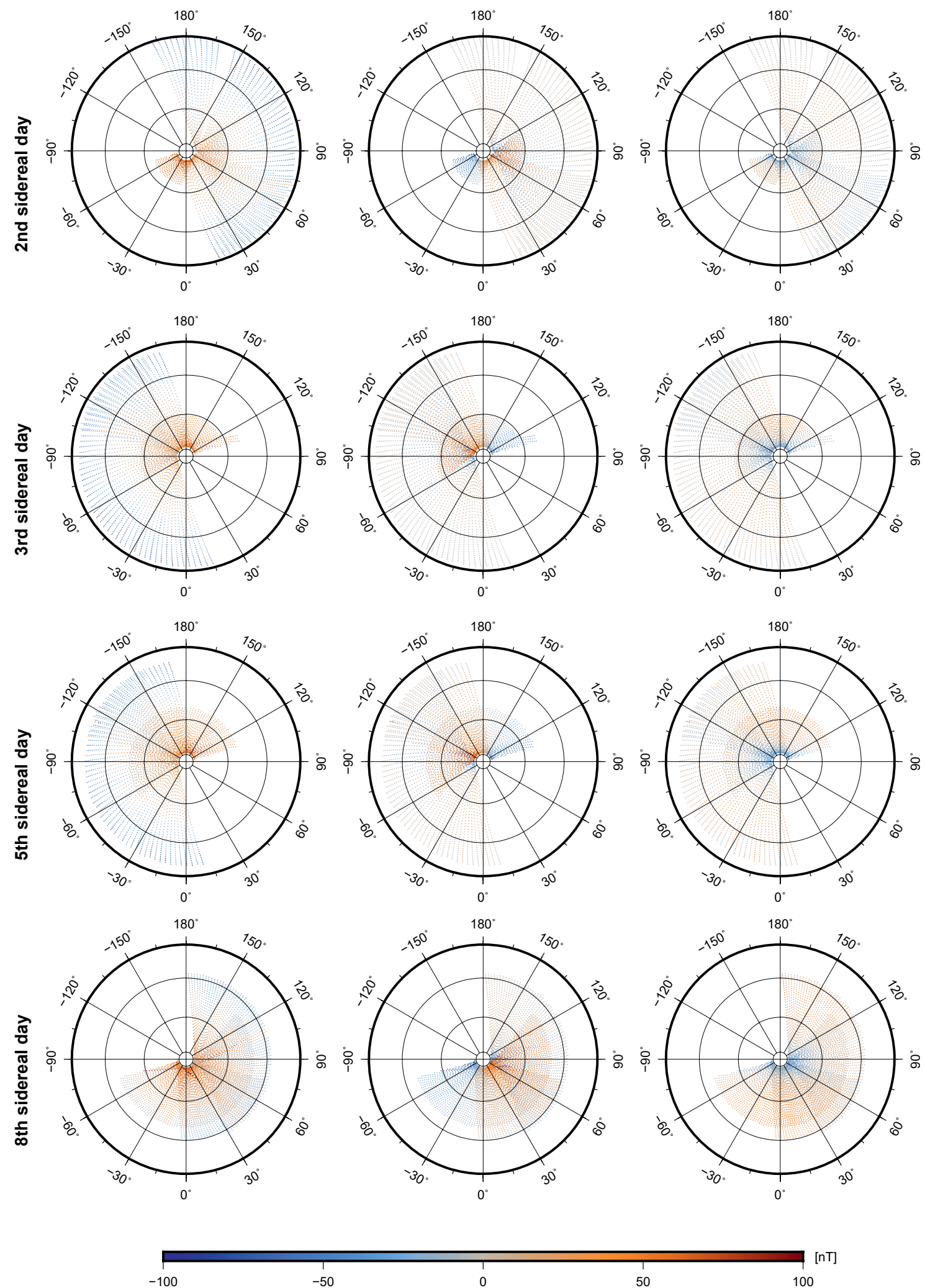

Figure 9. Equivalent maps as in Figure 8, but residuals are displayed in a coordinate system which is fixed with respect to Mercury, the MBF coordinate system. From left to right are shown the components $\mathrm{B}_{\theta}, \mathrm{B}_{\phi}$ and $\mathrm{B}_{r}$, respectively. 

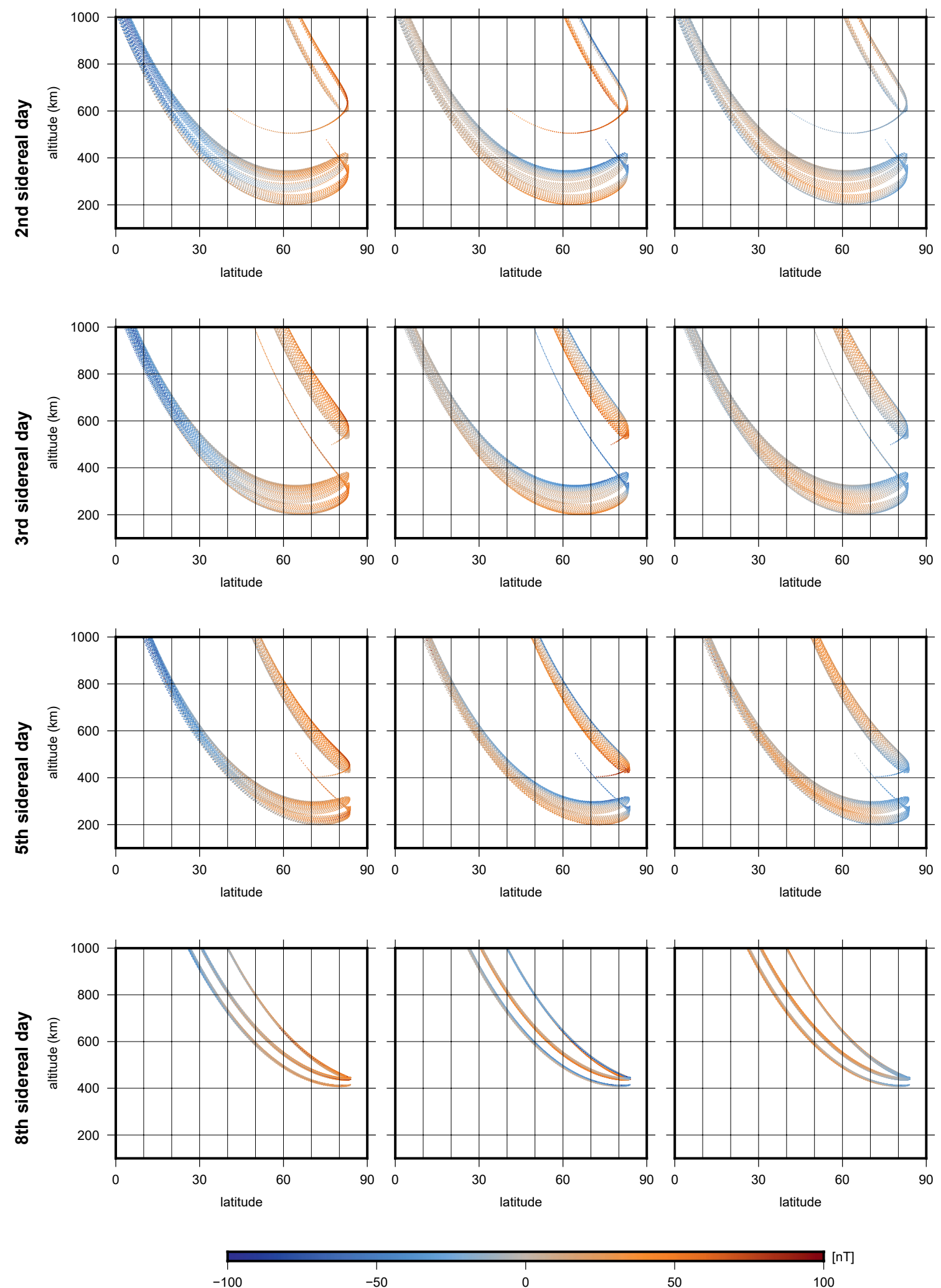

Figure 10. Residuals are shown as a function of latitude and altitude. From left to right the components $\mathrm{B}_{\theta}, \mathrm{B}_{\phi}$ and $\mathrm{B}_{r}$, respectively. 
Figure 1 of Busse, 1975) may explain the different signs of the non-zonal magnetic field structures. Cyclones/ anticyclones in the northern hemisphere correlate with convergence/divergence and concentrated/dispersed field, respectively (e.g., Olson \& Aurnou, 1999). Accordingly, in Figure 7 the flux patches B \& D may be concentrated by fluid downwellings associated with cyclones, while the positive non-zonal field (i.e., relatively weak field) in A \& C may be dispersed by fluid upwellings associated with anticyclones.

We interpret the latitude of these flux patches by comparing them to Earth's magnetic core field. Amit et al., 2011 quantitatively identified centers of geomagnetic intense flux patches. Their Figure 9 and our Figure C1 suggest that patch centers appear persistently at latitudes somewhat lower than that of the tangent cylinder. Analysis of the gufml historical geomagnetic field model (Jackson et al., 2000) reveals that patch latitudes are time-dependent, appearing from about $30^{\circ}$ latitude lower than the tangent cylinder until very close to it. However, in recent epochs when the field models are more reliable the patches reside less than $\sim 10^{\circ}$ lower than the tangent cylinder (Amit et al., 2011). This agrees with our analysis of a recent IGRF model (Thébault, Finlay, Beggan, et al., 2015). The latitude of the geomagnetic flux patches based on Figure $\mathrm{C} 1$ is approximately $8^{\circ}$ lower than that of the tangent cylinder. We conclude that based on the behavior of the geomagnetic field the offset between the patches and the actual co-latitude where the tangent cylinder intersects the CMB is roughly $\delta \theta \sim 10^{\circ} \pm 10^{\circ}$, that is, the patches reside in between the edge of the tangent cylinder to $20^{\circ}$ lower latitude.

Figure 11 (top) illustrates the classical tangent cylinder geometry with the addition of the effect of $\delta \theta$. This geometry is written as

$$
\sin \left(\theta_{c m b}-\delta \theta\right)=\frac{r_{i}}{r_{o}}
$$

where $r_{i}$ and $r_{o}$ are the radii of the inner core and the CMB, respectively. We assume that $\theta_{c m b}$ can be obtained from Mercury's non-zonal field and that $\delta \theta$ is similar to Earth's value. This allows to derive $r_{i}$, Mercury's inner core size. However, the presence of a stratified layer at the top of the core complicates this inference. When such a layer exists, the convective rolls concentrate flux at the base of the stratified layer, from which a skin effect may carry the signal to the CMB by diffusion (Christensen, 2006; Christensen \& Wicht, 2008). The presence of zonal flows in the stratified layer (Christensen, 2018; Olson et al., 2018) might complicate our inference of the core structure, though these flows would advect the magnetic flux patches in the east-west direction with a lesser impact on their latitudes which is the focus of our analysis.

Assuming that the signal propagation across the stratified layer is roughly radial, then Figure 11 (bottom) illustrates the relation between the colatitude of the patches $\theta_{c m b}$, the radius of the inner core $r_{i}$, and the radius of the base of the stratified layer $r_{s}$. Mathematically this relation is

$$
\sin \left(\theta_{c m b}-\delta \theta\right)=\frac{r_{i}}{r_{s}}
$$

which contains two unknowns, $r_{i}$ and $r_{s}$, and therefore cannot be uniquely determined. However, it provides a useful constraint and may be used to highlight various plausible scenarios for Mercury's internal structure.

To estimate Mercury's inner core size, we first estimate $\theta_{c m b}$ from Mercury's magnetic field model. The centers of intense flux patches B \& D (Figure 7) reside at about latitude $\sim 65^{\circ}$ north, or colatitude $\theta_{c m b}=25^{\circ}$. From the analysis of the geomagnetic field we further assume $\delta \theta=10^{\circ} \pm 10^{\circ}$. Substituting these values into (7) gives scenarios for Mercury's internal structure. Figure 12a presents the results for Mercury's inner core size $r_{i}$ and the thickness of the convective region is given in Figure 12b. Both are functions of the radius of the base of the stratified layer $r_{s}$, for three values of $\delta \theta$, which cover the considered range. Small values of $r_{s}$, which correspond to a very deep base of the layer (thick layer), give a very small inner core which would render the production of non-zonal features at the base of the stratified layer and hence the identification of a tangent cylinder effect to be impossible. Moreover, the magnetic Reynolds number scales with the convective shell thickness; if most of the core is stratified, a dynamo action is unlikely. Larger values of $r_{s}$, which correspond to a thinner stratified layer, give a thicker inner core with stronger dependence on $\delta \theta$. For a thin stratified layer of $\sim 50 \mathrm{~km}$, with $\delta \theta=0^{\circ}$ we find an upper bound $r_{i} \sim 850 \mathrm{~km}$ and a convective region of $1,160 \mathrm{~km}$, with $\delta \theta=10^{\circ}$ we find $r_{i} \sim 500 \mathrm{~km}$ and a convective region of $1,490 \mathrm{~km}$, whereas with $\delta \theta=20^{\circ} r_{i}$ is $\sim 180 \mathrm{~km}$ and a convective region of $\sim 1,830 \mathrm{~km}$. On the other hand, with a thick stratified layer of $\sim 1,600 \mathrm{~km}$ for all $\delta \theta$ the inner core size is smaller than $200 \mathrm{~km}$ and the respective size of the active dynamo region is less than $400 \mathrm{~km}$. 

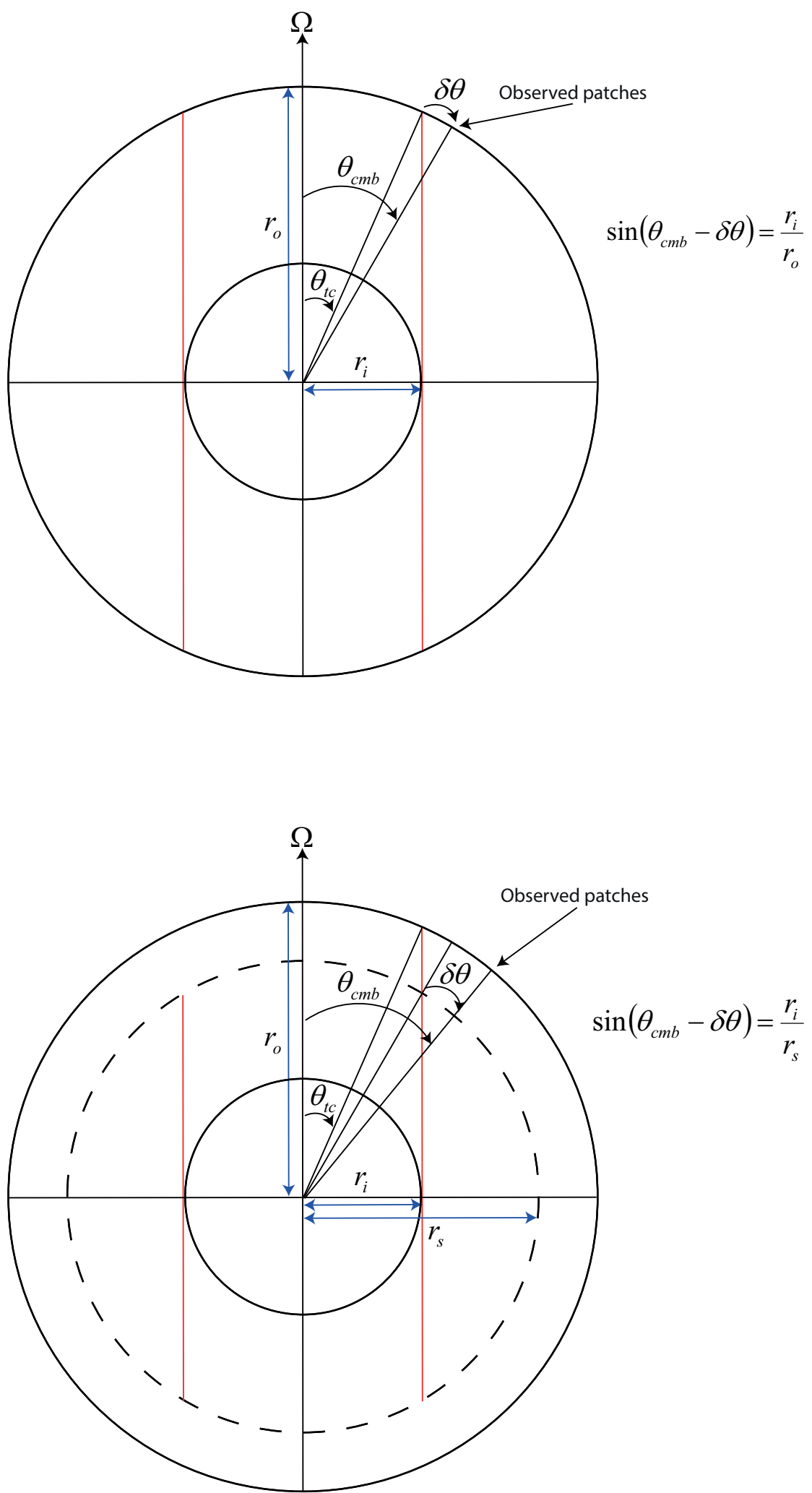

Figure 11. Schematic illustrations of the geometry of the tangent cylinder effect without (top) and with (bottom) a stably stratified layer. $\theta_{c m b}$ is the colatitude at which the tangent cylinder crosses the core surface and $\delta \theta$ s the latitudinal distance between the center of the magnetic flux patches and $\theta_{c m b}$. We discuss the range of $\delta \theta$ in the text. 

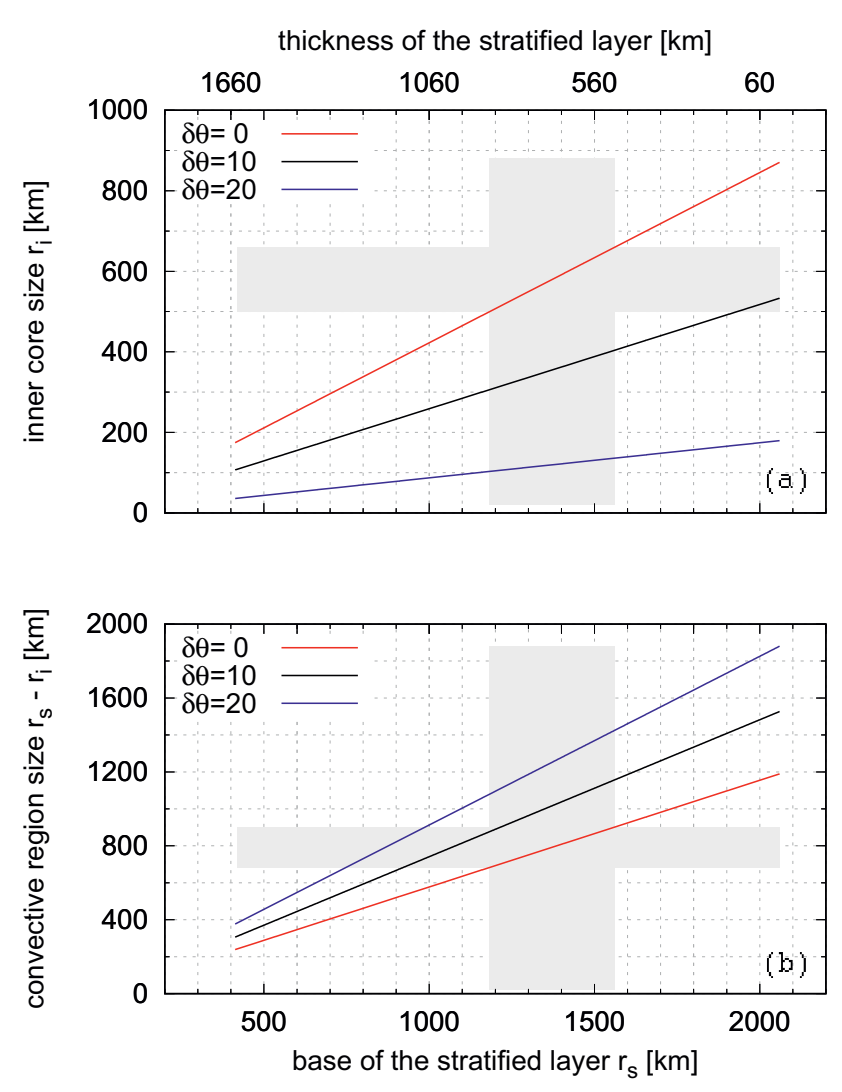

Figure 12. Mercury's inner core size $r_{i}$ (a) and the thickness of the convective region (b) versus the radius of the base of the stratified layer $r_{s}$ (or the layer thickness, see top horizontal scale), for three values of $\delta \theta$ (see legend). Gray areas indicate our proposed ranges for inner core size, thickness of the stratified layer, and the convection region size as discussed in Section 5.
In the discussion section we elaborate on the consequences of the latitude of magnetic flux patches on inferring the internal structure of Mercury.

\section{Discussion}

Figure 2 can be directly compared to the results of a resolution analysis by Uno et al., 2009. The resolution of our preferred model is certainly higher than the resolution of their inversion results. This is mainly because of the wider spatial coverage during the MESSENGER main mission (2011-2015) than during the three flybys of Mariner-10 and the one MESSENGER flyby in 2008. However, Uno et al., 2009 concluded that a realistic resolution up to spherical harmonic degree 10 can be obtained from the flyby data. We consider this as an optimistic view, as it (implicitly) assumes that model parameters with a small but nonzero resolution can be resolved by the inversion. In this respect, we are cautious in choosing a minimum resolution $R_{\min }$, which would be relevant for robust results. We find that a value of $R_{\min } \geq 0.4$ is a diligent choice for a required minimum resolution. The model solutions 1,2 , and 4 largely meet this criterion, and maps of these models are in good agreement when truncated to spherical harmonic degree $l=8$. Small-scale structures along the magnetic equator, particularly those seen in Models 1 and 2 , arise from spherical harmonic degrees $l \geq 6$.

The tangent cylinder effect is expected to hold when the dynamic is dominated by rapid rotation effects. This is the case in Earth's core (e.g., Aubert, 2013; Jault, 2008; Long et al., 2020). However, the dynamical regime in Mercury's liquid core is largely uncertain due to the unknown convection vigor there. To reproduce the magnetic equator offset of Mercury's field, the dynamo models of Cao et al., 2014 exhibit a superposition of two unstable columnar convection modes in rapidly rotating spheres, whereas the models of Takahashi et al., 2019 contain an antisymmetric flow component. Overall, caution is required when considering our results which would be valid only if rapid rotation effects govern Mercury's core dynamics. Bearing this in mind, our inference of the inner core size could provide insights to characterize the planet's internal structure and the dynamo action in its core. The morphology of Mercury's non-axisymmetric magnetic field that is shown in Figures $6 \mathrm{~b}$ and $6 \mathrm{~d}$ exhibits two high-latitude normal flux patches. The axisymmetric and non-axisymmetric parts of Mercury's magnetic field may be due to different processes. In this context it has been proposed that a stratified layer outside the dynamo region of Saturn leads to the axisymmetrization of its magnetic field (Stanley, 2010; Stevenson, 1982). Similar mechanisms are likely to be at work inside Mercury (Christensen, 2006; Christensen \& Wicht, 2008).

Apart from fluid downwelling and intense magnetic flux at the edge of the tangent cylinder, rapid rotation theory also predicts upwelling and weak (or even reversed) field at the poles. It has been suggested that polar minima are expected to emerge within the tangent cylinder and above the inner core due to core fluid upwelling (Bloxham et al., 1989; Gubbins \& Bloxham, 1987). Indeed, such polar minima are observed for Earth's magnetic field at the CMB (e.g., Jackson et al., 2000) and for numerical dynamo simulations (Cao et al., 2018; Olson et al., 1999). Our Mercury field models exhibit patches of high-latitude non-axisymmetric field, while polar minima are absent from our models which may have several reasons. Cao et al., 2018 found that the polar minima cannot be produced solely by axisymmetric, steady meridional circulations, that is, polar upwelling. Instead, they propose a tilted, or time-dependent, or fragmented upwelling scenarios, none of which will support and maintain minima at the poles at all times. Furthermore, the skin effect in the stratified layer most effectively diffuses non-dipole contributions if those small scales vary rapidly enough (Christensen, 2006). Figure C1 shows that the geomagnetic polar minima have a very large non-dipole part, as expected from reversed local polarity where the dipole field is strongest. These features are therefore expected to be strongly diffused by the skin effect. The non-dipolar contribution to the polar minima exceeds the non-dipolar contribution to the intense flux patches at the edge of the tangent cylinder (Figure C1), which may explain the survival of the latter in Mercury's field. We therefore 
hypothesize that while the stratified layer reduces the amplitude of the non-zonal field (which is indeed much weaker in Mercury than on Earth), the polar minima are almost diminished by the skin effect. In addition, if the top of Earth's core also has a stably stratified layer, as suggested by seismic, mineral physics and geomagnetic studies (e.g., Buffett et al., 2014; Kaneshima, 2018; Pozzo et al., 2012), why would polar minima survive the skin effect on Earth but not on Mercury? Recall that the stratified layer on Earth is in general debate (Amit, 2014; Irving et al., 2018; Konôpková et al., 2016; Lesur et al., 2015) or might be merely regional (Mound et al., 2019; Olson et al., 2017). In particular, the concentrated flux patches that characterize the geomagnetic field (Christensen et al., 2010) are difficult to reproduce with numerical dynamos that include a significantly thick or strong stratified layer (Gastine et al., 2020). Alternatively, it could be that the dependence of the secular variation timescales (Lhuillier et al., 2011) on spherical harmonic degree differs between Earth's and Mercury's fields.

Mercury's internal structure is still unresolved by geodetic analyses and there is a debate concerning the existence and possible size of a solid inner core. If a solid inner core exists, it was argued that its radius is likely to be smaller than 1,000 km (Cao et al., 2014; Dumberry \& Rivoldini, 2015; Peale et al., 2016; Van Hoolst et al., 2012). Based on estimates of Mercury's gravity field, tidal Love number, and pole coordinates, Mercury's inner core radius is in the range $883-1,026 \mathrm{~km}$ (Genova et al., 2019). However, these reported values of Mercury's inner core size are still questioned as estimates derived from a geodetic analysis of Mercury's orbital motion give a larger range of 370-1,200 km (from combining the first and third quartiles of Margot et al., 2018).

Based on the above estimates from geodetic analyses we consider Mercury's inner core size to be $r_{i}=500$ $1,000 \mathrm{~km}$. For $r_{i}=500 \mathrm{~km}$ and $\delta \theta=0^{\circ}$ Figure 12a gives a stratified layer thickness of $\sim 880 \mathrm{~km}$ which leaves $\sim 680 \mathrm{~km}$ for the convective region to maintain a dynamo. For $r_{i}=500 \mathrm{~km}$ and $\delta \theta=10^{\circ}$ the stratified layer thickness is $\sim 130 \mathrm{~km}$ and the convective region is $\sim 1,430 \mathrm{~km}$, while for $r_{i}=500 \mathrm{~km}$ and $\delta \theta=20^{\circ}$ a solution does not exist. Furthermore, an inner core size of $r_{i}=1,000 \mathrm{~km}$ is out of range for the considered $\delta \theta$ values (Figure 12a). Because the large-scale field of Mercury favors a substantial stratified layer and because large $r_{i}$ constrains $\delta \theta$ to small admissible values, we conclude that the inner core size tends towards the small end of the considered $r_{i}$ range.

The thickness of the stratified layer at the top of Mercury's core is also unknown. Smith et al., 2012 suggested that a $200 \mathrm{~km}$ thick and solid FeS-layer at the interface of a silicate mantle and the metallic core may explain the planet's moment of inertia. However, this setup was questioned by Hauck et al., 2013 who derived models without an FeS-layer to reproduce the gravity field observations and libration data. The thickness of such a layer depends on the available sulfur and its solubility in the metallic core determined by the widely unknown core temperature and reduction conditions (Hauck et al., 2013). In most numerical dynamo simulations that attempt to explain observations of Mercury's magnetic field, a thick layer is assumed from several hundred km (e.g., $600 \mathrm{~km}$ in Christensen, 2006; Christensen \& Wicht, 2008) up to half the core radius (Takahashi et al., 2019). The stratified layer weakens and diffuses the non-axisymmetric field via a skin effect, which could explain its low intensity and dominant axisymmetry.

Considering a stratified layer thickness of 500-1,000 km (or $r_{s}=1,560-1,060 \mathrm{~km}$ ), we obtain $r_{i} \sim 660-90 \mathrm{~km}$ and a dynamo region of $\sim 1,420-680 \mathrm{~km}$, respectively, with ranges corresponding to the different $\delta \theta$ values (Figure 12). The small inner core scenario (with $\delta \theta=20^{\circ}$ ) seems unlikely to produce a detectable tangent cylinder effect. We therefore favor again the solutions for low $\delta \theta$, which correspond here to inner core sizes of $\sim 660-450 \mathrm{~km}$ and convective region sizes of approximately $900-680 \mathrm{~km}$.

In summary, after consideration of the above detailed constraints, our analysis suggests the ranges of 500-660 km for the inner core size and 880-500 km for the thickness of the stratified layer below the CMB, respectively (see gray areas in Figure 12).

\section{Conclusion}

In this study, we investigate the morphology of Mercury's magnetic core field and the smallest possible spatial scales that can be resolved from the MESSENGER measurements. Our spherical harmonic analysis demonstrates that features of the steady magnetic core field of spherical harmonic degree $l=8$ can be robustly resolved; higher spherical harmonic degrees are likely aliased by undetermined magnetic signatures. Different data selection schemes to avoid magnetic signatures due to field aligned currents in the cusp region and to reduce effects of 
magnetospheric disturbances do not alter the modeling results significantly. Our magnetic field models consistently suggest the existence of non-axisymmetric features of the Mercury's core field. Although this consistency may be due to the modeling technique and the set of priors, the resolution analysis indicates that these features are largely supported by the data.

For the first time, Mercury's non-axisymmetric core field is identified and studied to infer the internal structure of its core. We find non-axisymmetric flux patches at high northern latitudes. We interpret these features as the signature of convective columns adjacent to the inner core tangent cylinder. The deviation from axisymmetry introduced by these patches is far less pronounced than at Earth's geomagnetic field due to the masking by Mercury's dominant axisymmetric field.

We take advantage of the mean latitude of these two patches to constrain Mercury's internal structure. We establish a relation between the inner core size and the thickness of the stratified layer below the CMB as a function of the latitude of the magnetic flux patches. While various combinations of these two quantities are possible, a combined interpretation of our results and those from geodetic analyses limits the range of the inner core radius to $\sim 500-660 \mathrm{~km}$. Accordingly the stratified layer thickness is $\sim 880-500 \mathrm{~km}$, leaving $\sim 900-680 \mathrm{~km}$ for the convective dynamo region, respectively. Furthermore, our results favor little (if any) shift between the locations of magnetic flux patches and the tangent cylinder at the top of the dynamo region, in apparent contrast to the offset observed at Earth's core.

Finally we emphasize that our analysis is based on a data set of the MESSENGER mission over the northern hemisphere only. This puts limits on the magnetic field models and the inferences concerning Mercury's internal structure. The future Bepi-Colombo mission will unravel these details of Mercury's magnetic core field.

\section{Appendix A: Alternative Data Selection Schemes}

To validate the internal (core) origin of our magnetic field models, we devise three additional data selection schemes to exclude magnetic signatures of field-aligned currents (schemes I + II) and to assess the influence of different disturbance conditions (scheme III). These schemes can be considered to be more rigorous than the scheme on which our study is based. The additional schemes are:

I) selection of night-time data between 8p.m. and 4a.m. local time,

II) selection of night-time data between $8 \mathrm{p} . \mathrm{m}$. and 4a.m. local time, and on ascending orbits only consider $B_{r}$ for latitudes $\geq 80^{\circ}$ North, and on descending orbits only consider $B_{r}$ for latitudes $\geq 70^{\circ}$ North.

III) selection of night-time data between 7p.m. and 5a.m. local time and with respect to different magnetic disturbance conditions using a magnetic disturbance index (MA) suggested by (Anderson et al., 2013; Philpott et al., 2020).

The first scheme avoids the dawn and dusk sections and their current systems, first by limiting the data selection to a smaller section of night-time, than the selection used in the main manuscript. This seems to be required as some lobes of the Birkeland currents extend to $4 \mathrm{a} . \mathrm{m}$. and 8p.m. local night-time. The second scheme is derived from Figure 3 of Anderson et al., 2014, see Figure A1. The large gray areas in both panels of this Figure cover local day side and dawn-dusk sections, where data show contributions from external magnetospheric fields (as the magnetosphere may touch Mercury's surface) and Birkeland currents. Those data are discarded for our field modeling. For schemes (I) \& (II), we only select data from un-shaded areas.

The first selection scheme leaves some field-aligned current features unaccounted for, particularly at high latitudes (blue and red patches in Figure A1). Effects of these features may average out over the mission's lifespan in a coordinate system rotating with the planet. Note, that Mercury rotates in a local time frame with a period of 156 (terrestrial) days, and MESSENGER with a period of 88 days.

To further account for the remaining magnetic signatures of Birkeland currents at high latitudes (blue and red patches in Figure A1), we select only measurement of the radial field component in scheme (II). The reason is that 

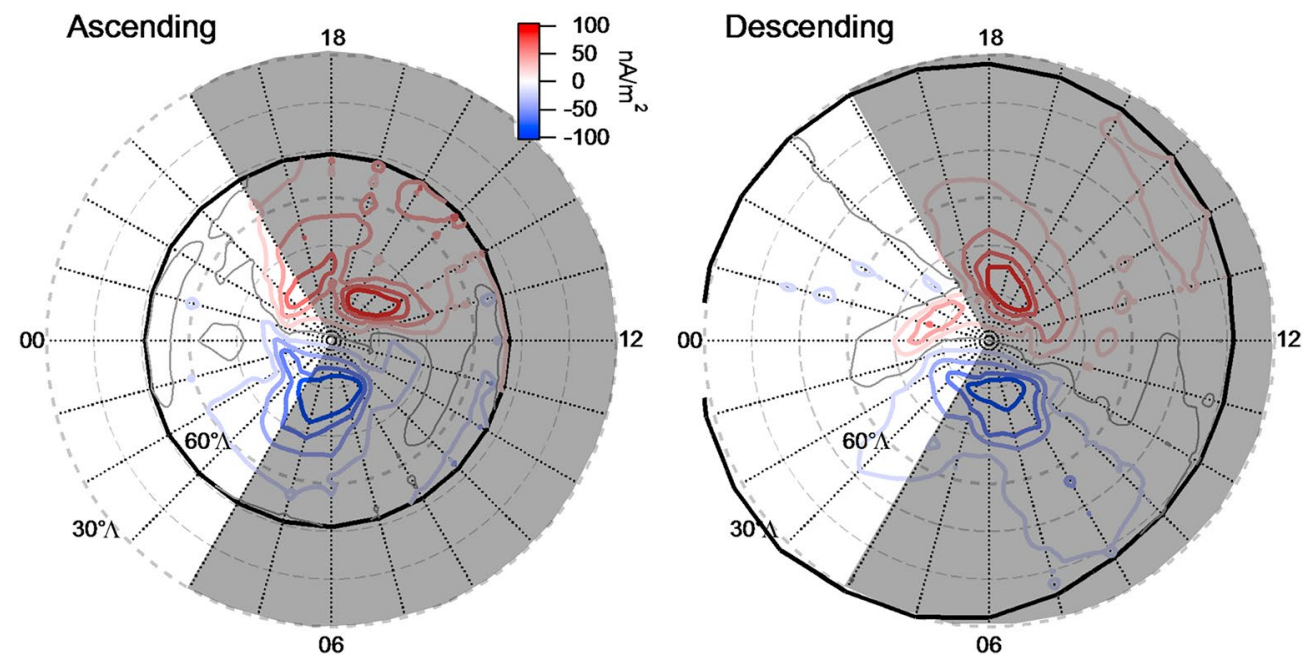

Figure A1. Distribution of Birkeland currents at Mercury with respect to local time, on the left for ascending orbits, and descending orbits at the right. The sun is towards 12:00, dawn at 18:00, midnight at 00:00, and dusk at 06:00, respectively. The Figure is adopted from Figure 3 of Anderson et al., 2014 and gray shaded areas mark local times from 04:00 to 20:00 day-time. Data from these shaded sections are not used for the magnetic field modeling. The black line encircles the region over which data were analyzed by (Anderson et al., 2014). (Figure courtesy of B. Anderson)

field-aligned currents close to the North pole are aligned with the field in the radial direction and flow in the radial direction. Therefore, their induced/generated magnetic field is perpendicular, that is, in horizontal directions $B_{\theta}$ and $B_{\phi}$, with no magnetic signatures in the radial component (Anderson et al., 2014, 2018). The application of the second scheme should thus remove every magnetic signature of field aligned currents.

Scheme (III) is set up to assess the robustness of our internal magnetic field model and its features with varying magnetic activity (disturbances) in Mercury's magnetosphere. We apply three levels of magnetic activity: (a) MA $<60 \%$-moderately disturbed, (b) MA $<30 \%$-quiet and (c) MA $<10 \%$-very quiet conditions, respectively. We provide Table A1 that lists model characteristics, to complete our analyses of different data selection schemes.

Figure A2, Figure A3, Figure A4, and Figure A5 are based on models obtained from the different data selection schemes, for which we employ the same prior as for Model 4, for example, Norm 4, and choose the same damping parameter, except for Figure A6, where we plot a solution that is closer to the optimum (knee of the trade-off curve). Maps a \& c of these Figures are very similar to those of Figure 6 . This is also the case for maps $b \& d$ of Figures 6 and A2-A5, where the four patches around the planet's North pole are almost equally displayed. The morphology of the non-zonal radial field in maps b \& d of Figure A6 varies slightly for very quiet magnetospheric conditions. However, this can be explained by the significantly reduced and poorer data set, which does not allow to resolve the very details of the internal magnetic field as indicated by the number of resolved model parameters, see also Table A1. We note, that a large axial quadrupole-dipole ratio relates to a larger misfit for data selected during very quiet magnetospheric conditions, see Table A1. The amplitude of residuals of the solution for III C is enhanced by $14 \mathrm{nT}$ (about 75\%) when compared to the optimal solution III $\mathrm{C}^{\star}$.

Figure A7 shows the resolution curves of the models based on different data selection schemes. The solution III $\mathrm{C}$ has the lowest resolution of all models. Misfit and rms field intensity indicate that this solution is not optimal. Relaxing the spatial prior by weakening the damping parameter $\lambda_{s}$ by a factor of 8 provide a near optimal solution with lower misfit and higher resolution. Taking this into account, then all models show common resolution curves that are similar to that of model 4 (Figure 2), but differ in their numbers of resolved parameters. 
(a)

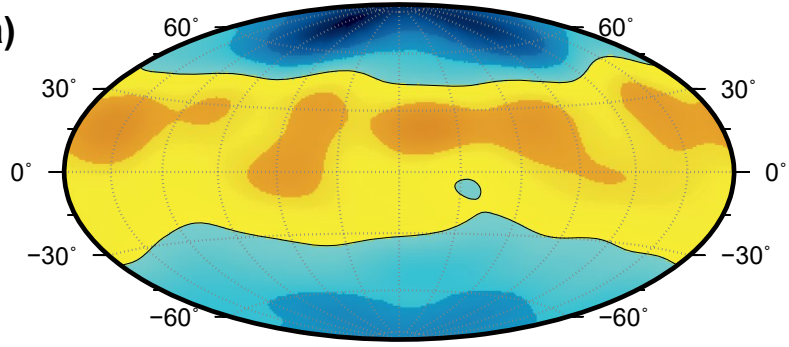

(c)
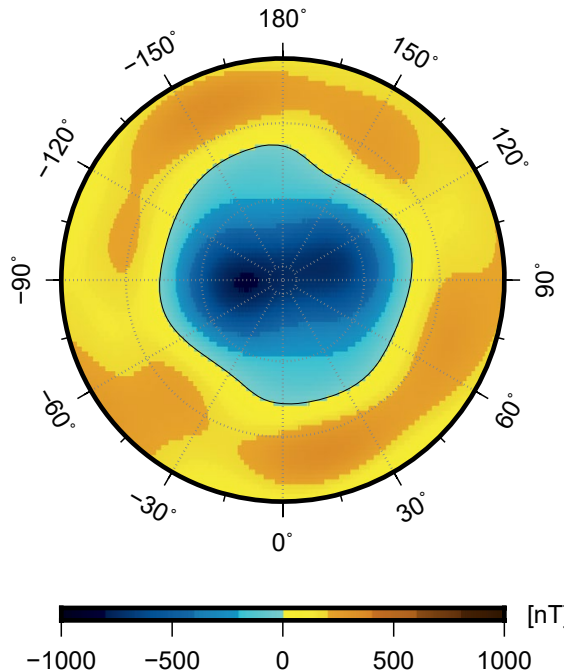
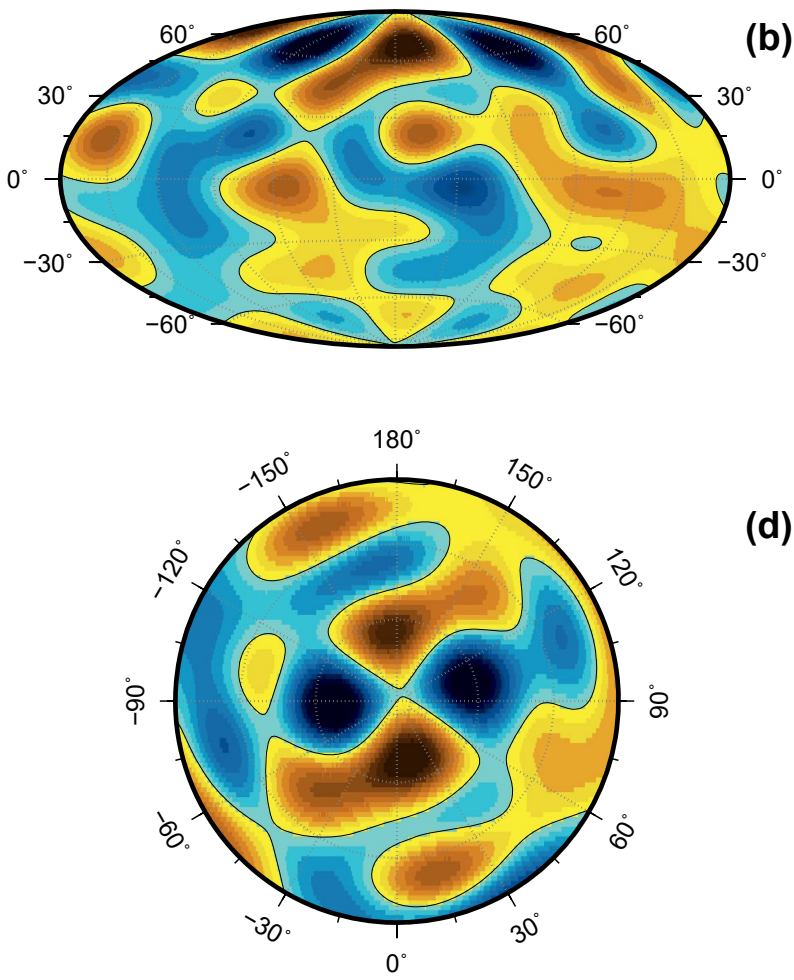

(d)

Figure A2. Same maps as Figure 6 derived from data selection scheme I. 
(a)

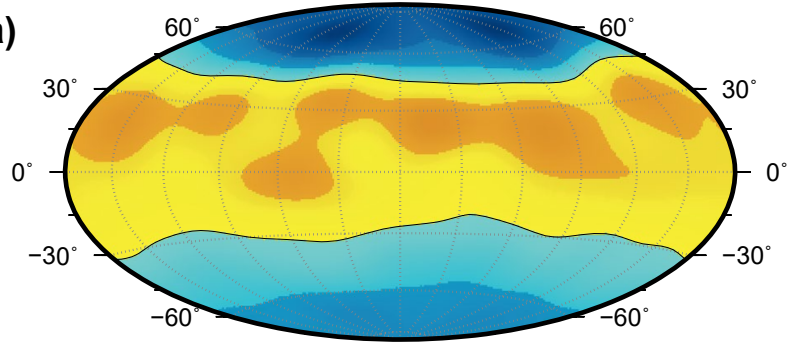

(c)
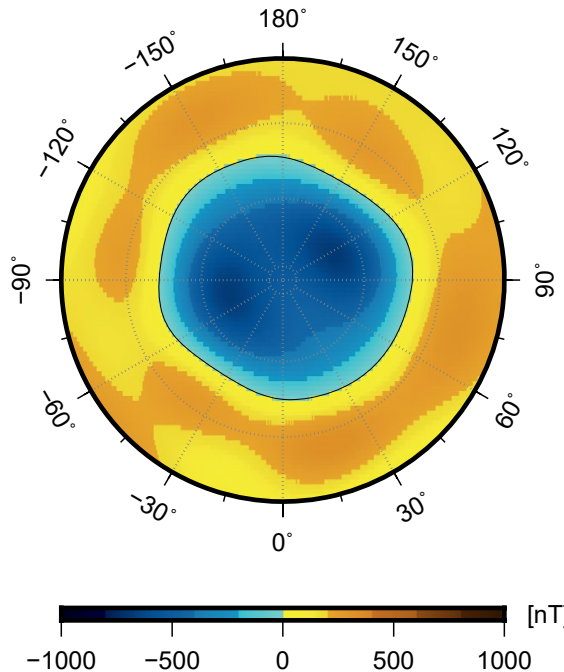
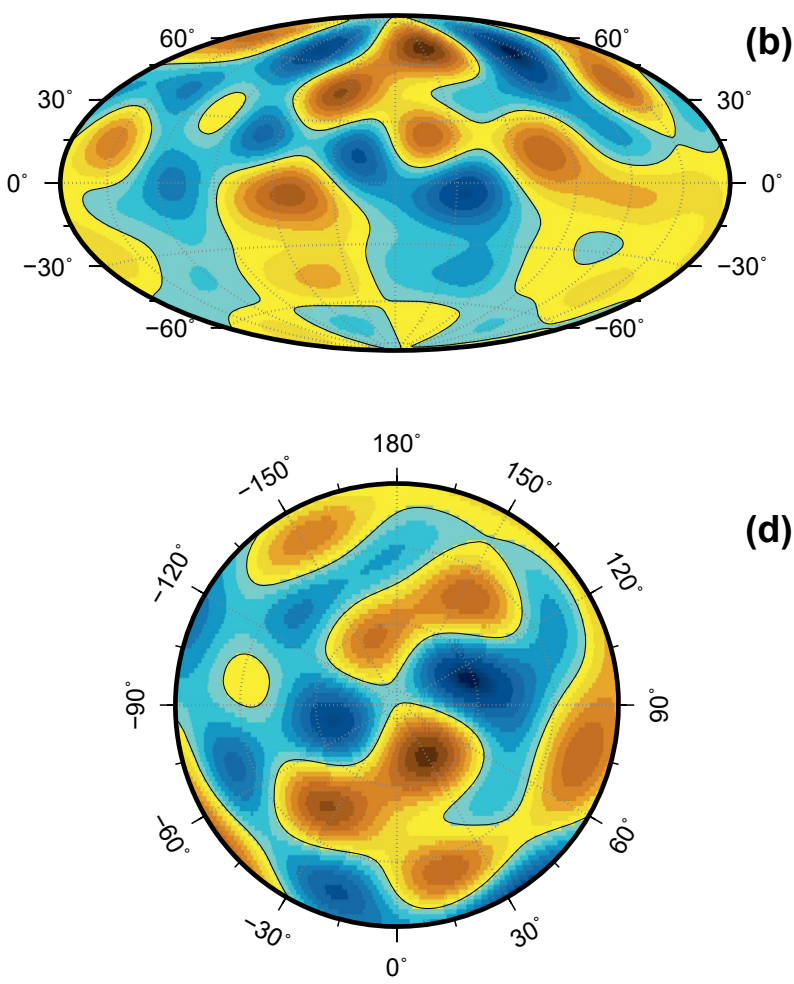

(d)

Figure A3. Same maps as Figure 6 derived from data selection scheme II. 
(a)
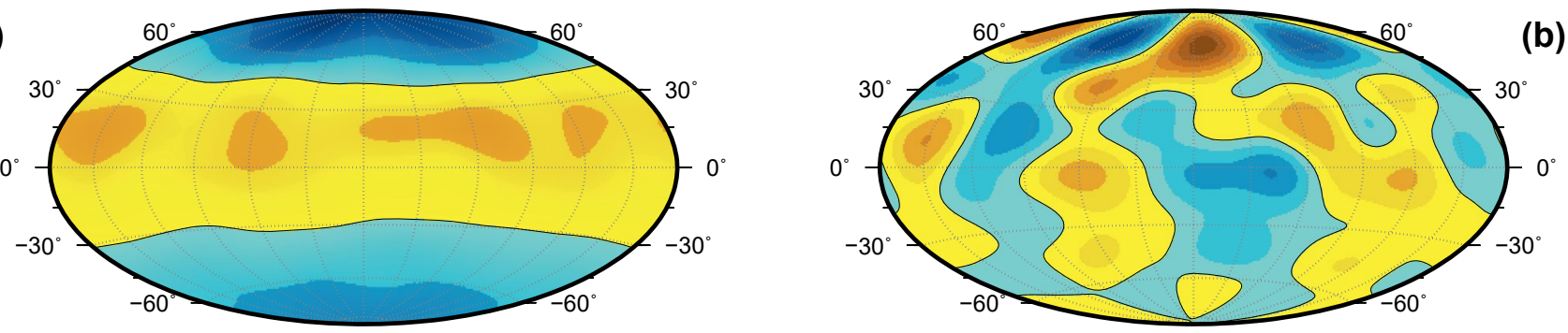

(c)
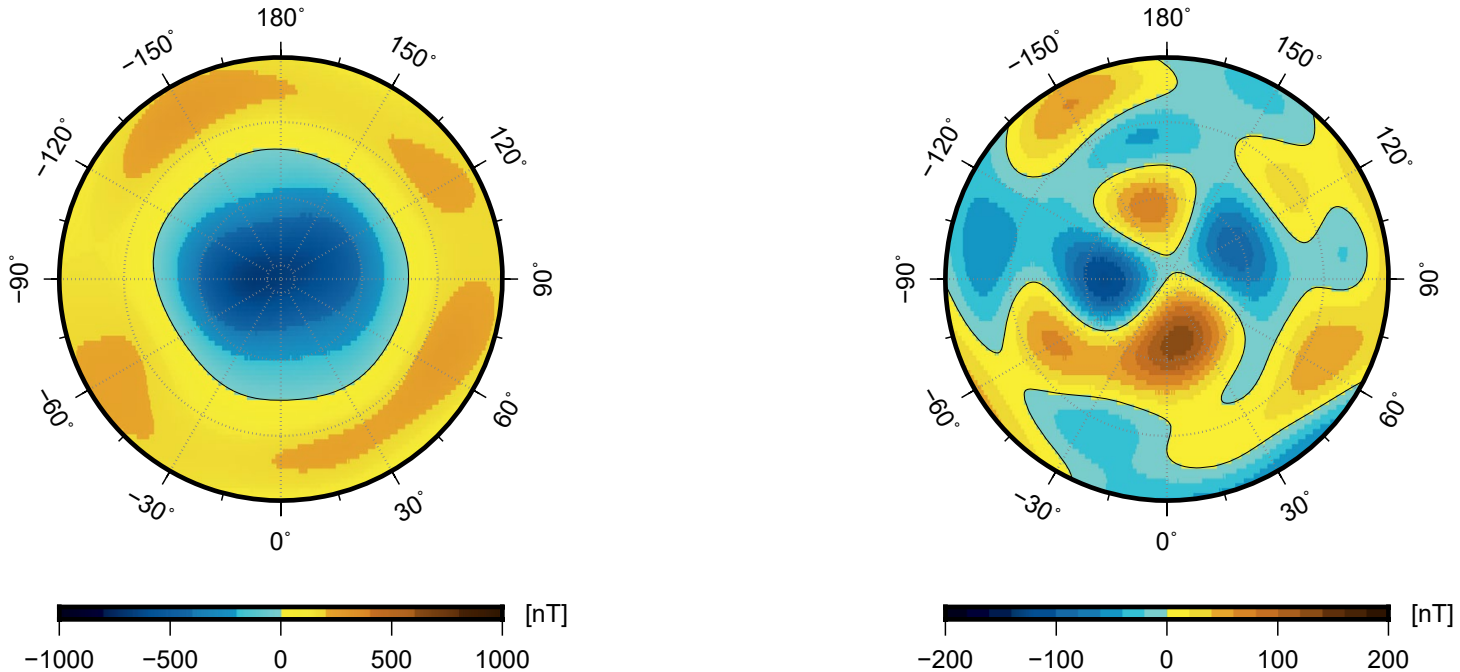

(d)

Figure A4. Same maps as Figure 6 derived from data while the magnetic activity index MA $<60$, that is, III A. 
(a)
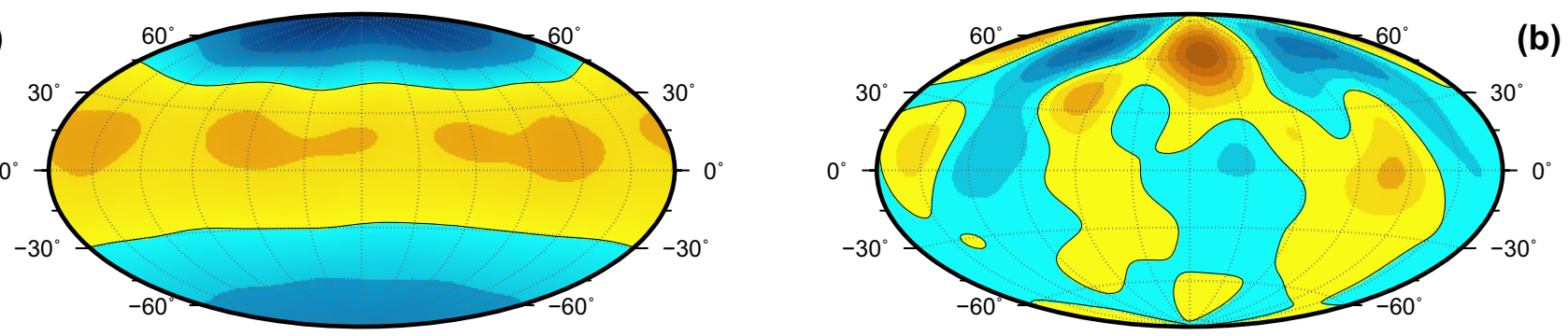

(c)
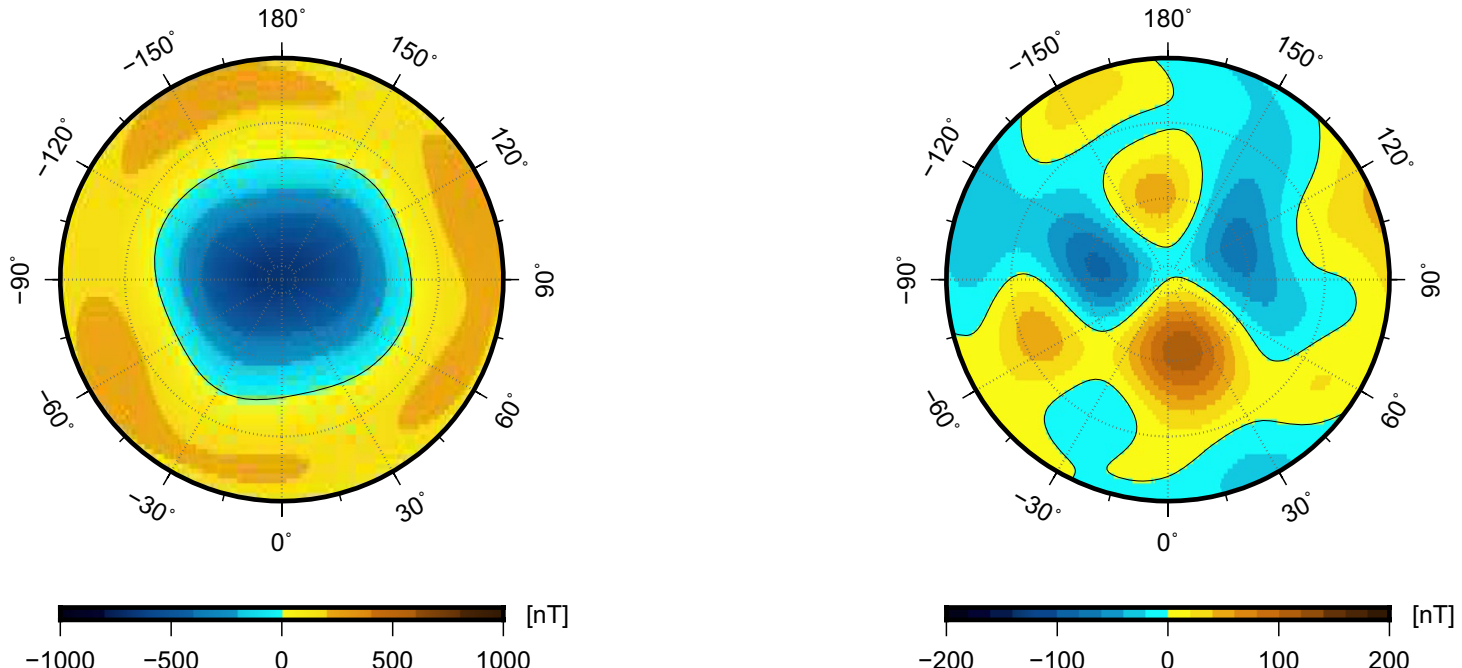

(d)

Figure A5. Same maps as Figure 6 derived from data while the magnetic activity index MA $<30$, that is, III B. 
(a)

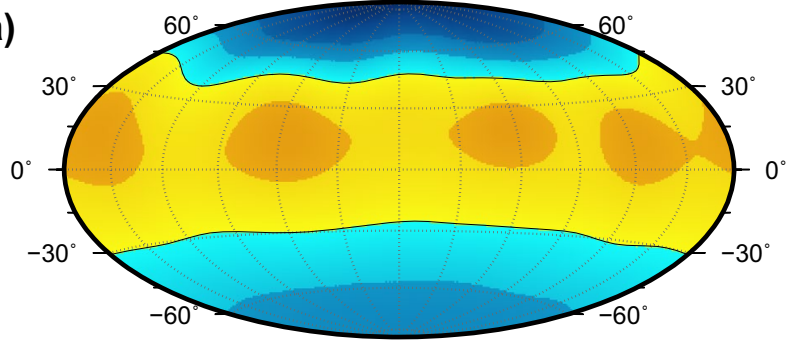

(c)
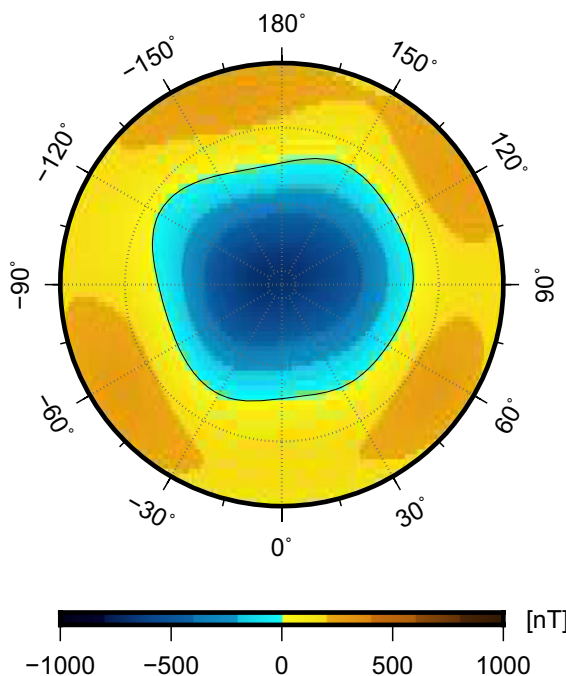

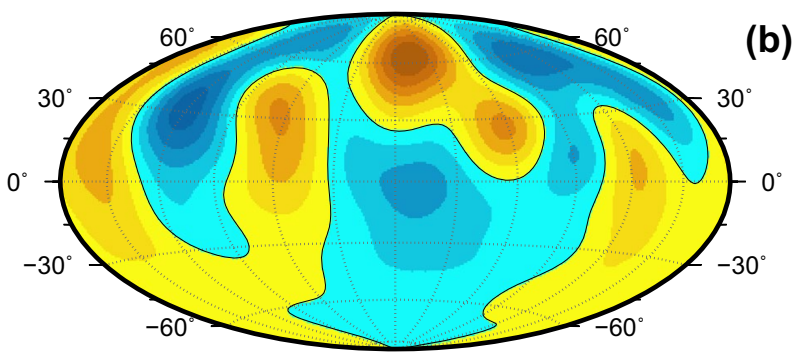

(b) $0^{\circ}$ $-30^{\circ}$

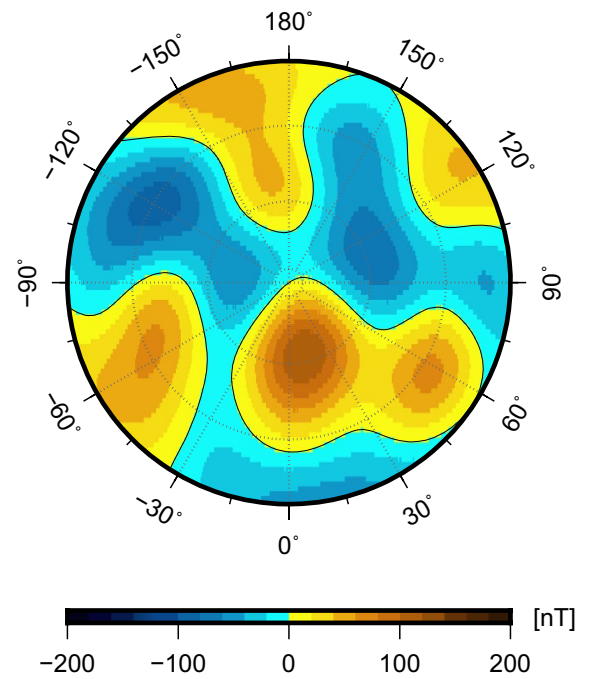

(d)

Figure A6. Same maps as Figure 6 derived from data while the magnetic activity index $M A<10$. Plotted is the map of the optimal solution $M A<10$, that is, III $C^{\star}$.

The first main outcome of the analyses of different data selection schemes is that there are no significant differences between maps of Figures 6, A2 and A3. In Figure A2, features are stronger than in Figure 6, but both Figures present the same morphology. In Figure A3, though general characteristics of the field are unchanged, there is a slight change of the field morphology. The change is certainly caused by the selection of only the radial field component over the North pole. A consequence of using only radial components, is that the separation of internal and external field contributions is less robust (Lesur et al., 2008), and therefore may cause a slight change of the field morphology. However, we consider these maps of the three different models to agree with each other, that

Table A1

Global Characteristics of the Field Models Derived Using Different Data Selection Schemes I and II Compared to the Values of Model 4. the Damping Parameter of Solution III $C^{\star}$ is $\lambda_{s} 1.0 \times 10^{6}$

\begin{tabular}{|c|c|c|c|c|c|c|c|}
\hline Selection scheme & I & II & III A & III B & III C & III $C^{\star}$ & Model 4 \\
\hline Vector triplets & 1109655 & 873092 & 786899 & 393010 & 133346 & 133346 & 1360051 \\
\hline rms misfit (nT) & 25.75 & 22.47 & 22.87 & 21.26 & 34.69 & 20.07 & 26.39 \\
\hline Trace of R-Matrix & 51 & 56 & 51 & 44 & 21 & 38 & 57 \\
\hline rms field intensity (nT) & 337.53 & 335.15 & 331.9 & 321.9 & 219.0 & 312.89 & 335.24 \\
\hline$g_{1}^{0}(\mathrm{nT})$ & -223.0 & -220.5 & -218.5 & -209.1 & -132.6 & -219.4 & -221.5 \\
\hline$g_{2}^{0} / g_{1}^{0}$ & 0.29 & 0.30 & 0.29 & 0.33 & 0.44 & 0.29 & 0.28 \\
\hline
\end{tabular}




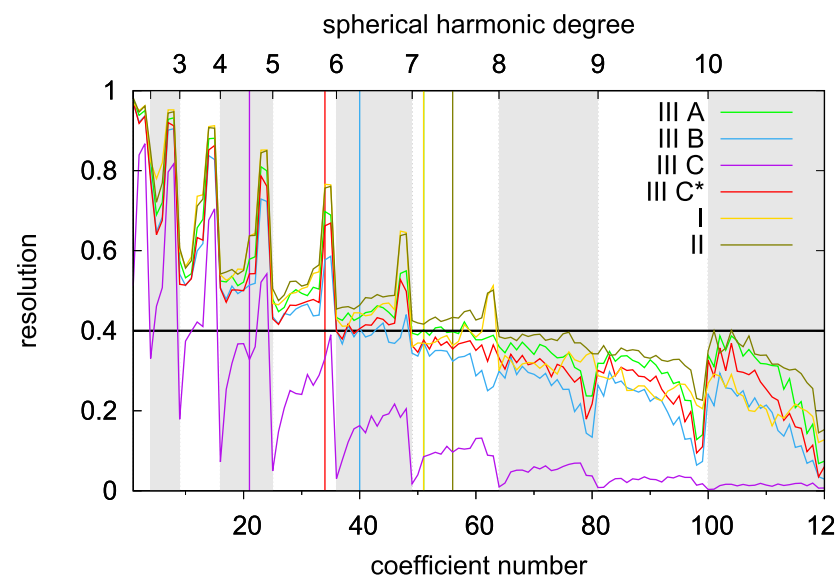

Figure A7. Resolution curves of models derived with different data selections. Same arrangement as in Figure 2.

is, the selection of night time data leads to robust models of Mercury's internal magnetic field that is common to different settings of the night time data selection.

The very similar results for the internal magnetic field models based on different data selection schemes strongly suggests that the described non-zonal radial magnetic field features in our study are of internal origin. Therefore, our interpretations upon Mercury's core are not biased by un-modeled external magnetic fields nor by magnetic signatures of Birkeland currents.

The second main outcome relates the robustness of our internal magnetic field modeling with respect to different magnetic activity conditions of Mercury's magnetosphere. We find that our representation of Mercury's internal magnetic field does not depend significantly on the selection of data with respect to the magnetic activity measured by the disturbance index (Anderson et al., 2013; Philpott et al., 2020). Variations of the field morphology between different activity levels can be explained by the varying spatial resolution of the field models, as significant fewer data are available for very quiet conditions than for conditions of enhanced magnetic activity.

\section{Appendix B: Covariance Analysis}

We study the robustness of our inversion results by analyzing the resolution matrix. Yet, another way to estimate formal uncertainties of the results is by analyzing the covariance matrix, which is given by

$$
\mathbf{C}=\hat{\sigma}^{2}\left(\mathbf{A}^{\top} \mathbf{C}_{\mathbf{e}}^{-1} \mathbf{A}+\lambda_{s} \mathbf{C}_{\mathbf{m}}^{-1}\right)^{-1}
$$

where $\hat{\sigma}^{2}$ is the misfit between model and data. These errors are formal, as they represent the uncertainty in the model subject with respect to the constraint and may be invalidated by false observations or by inappropriate prior information. It does not contain that part of the uncertainty, which is related to a trade-off in resolution, when combinations of parameters have the same effect on the fit to the data (Bloxham et al., 1989). In this sense, diagonal elements of $\mathbf{C}$ are the formal variances of the model parameters, and the off-diagonal elements are the formal covariance between individual model parameters.

In Figure B1 the diagonal elements of the four models covariance matrices are shown. The largest variances are found for coefficients of spherical harmonic degrees 2 and 3, that is, $g_{2}^{1}$ and $g_{3}^{1}$. Generally, the formal error is small, and depends on the strength of the damping parameter $\lambda_{s}$; weaker damping enhances the formal error.

Figure B2 shows the scaled covariance matrix elements of the four models. These matrix elements are scaled by the variances of the diagonal elements. This scaling procedure enhances covariance structures of the non-diag- 


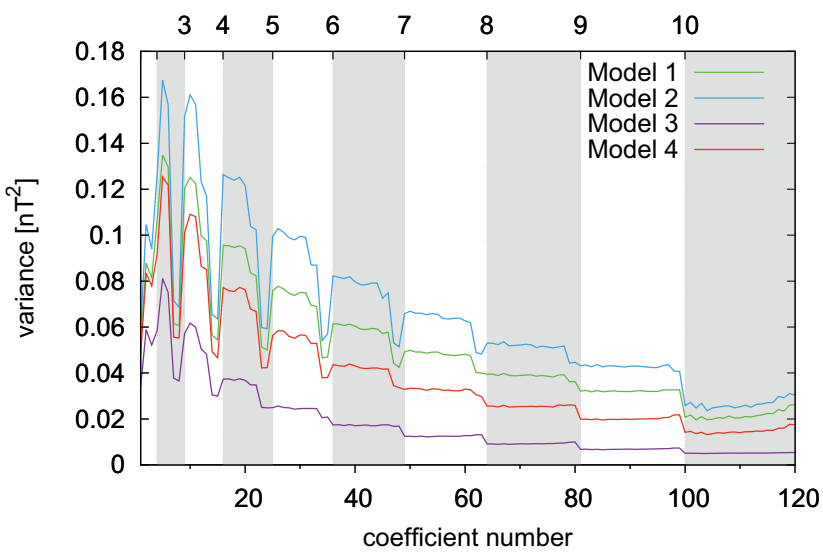

Figure B1. Diagonal terms of the four models covariance matrices.

onal elements. The scaled covariance matrices of Models 1,2, and 4 are very similar in their off-diagonal structures, whereas those structures are rather faint in the covariance matrix of Model 3. Large negative covariance (blue) occur between $g_{2}^{0}$ and coefficients of the first spherical harmonic degree (lower left corner of each plot). This trend continues between coefficients of consecutive spherical harmonic degrees for example, $C\left(g_{3}^{1}, g_{4}^{1}\right)$, which leads to "parallel" off-diagonal structures. Positive covariance structures are less pronounced and occur between coefficients with two spherical harmonic degrees differences for example, $C\left(g_{2}^{0}, g_{4}^{0}\right)$. These structures are mostly visible for Models 1 and 2. The cause of these off-diagonal structures is the uneven hemispherical data distribution. 
(a)

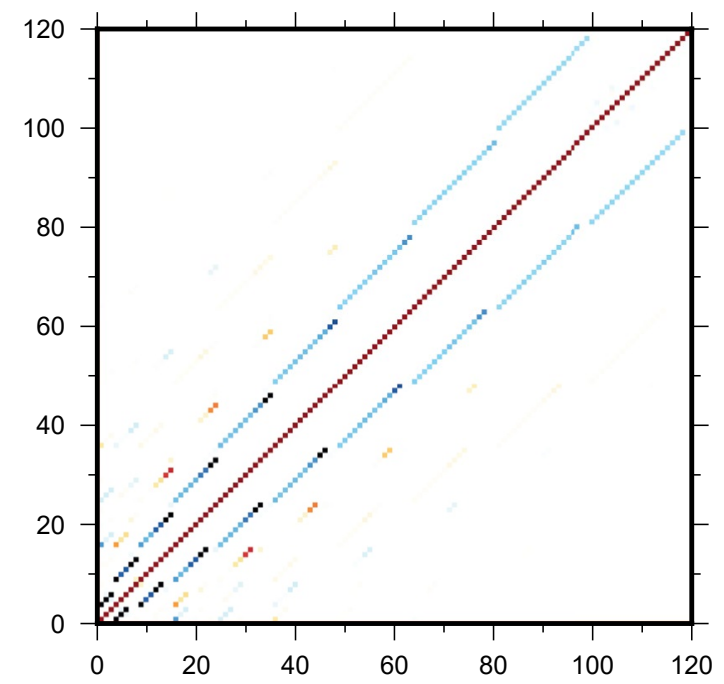

(c)

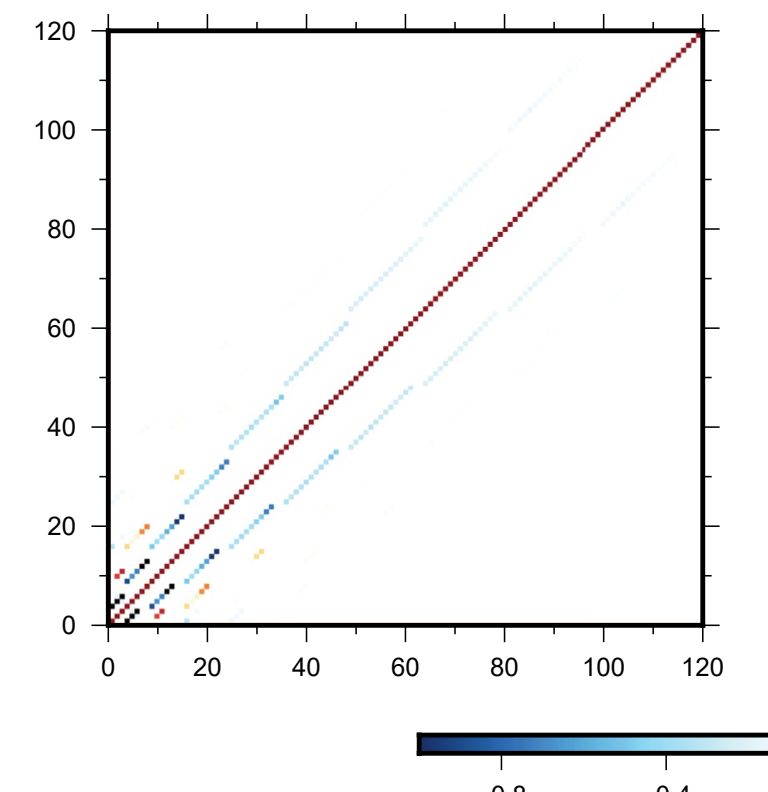

$-0.8$

$-0.4$

Figure B2. Covariance matrix elements of the four model solutions. Elements are scaled by the covariance of the diagonal elements $C\left(g_{n}^{n}, g_{n}^{n}\right)$ to enhance the visibility of the non-diagonal terms. a, b, c and d refer to matrices of Models 1, 2, 3 and 4, respectively.

\section{Appendix C: Earth's Core Field}

Figure $\mathrm{C} 1$ shows the radial geomagnetic field component, its non-axisymetric part and its non-dipolar part at Earth's core surface in the year 2015. The maps are based on the $12^{\text {th }}$ International geomagnetic reference field (Thébault, Finlay, Beggan, et al., 2015). The model was truncated at spherical harmonic degree $L_{\text {int }}=10$. The maps show also the projection of the inner core tangent cylinder on the CMB. 


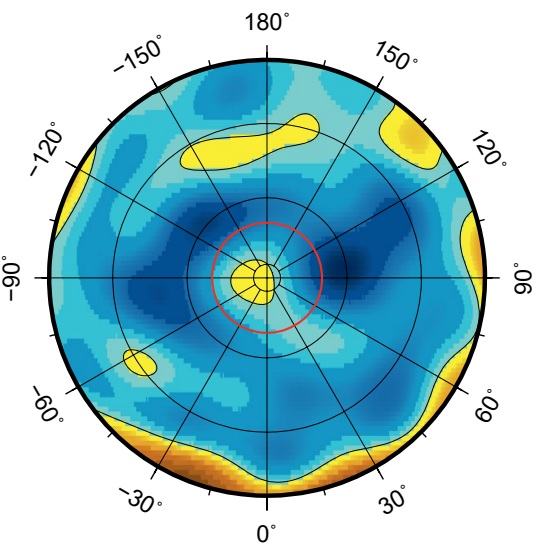

(a)

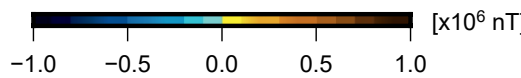

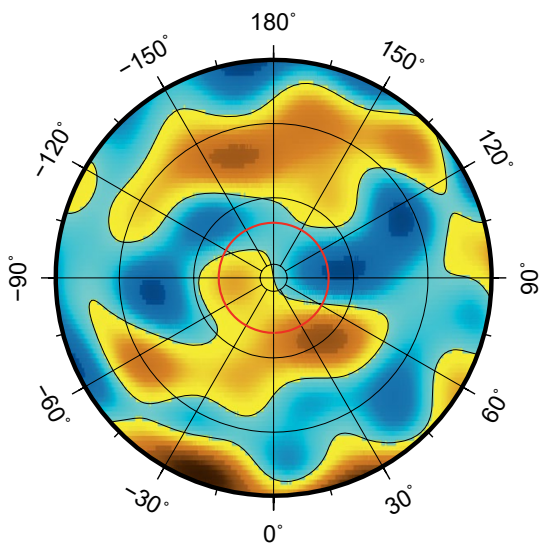

(b)

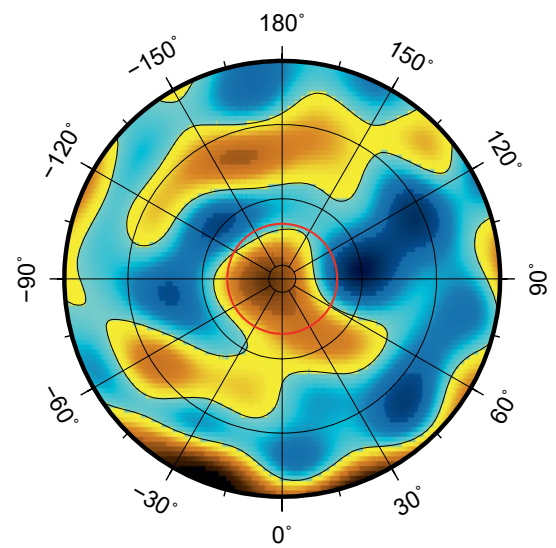

(c)

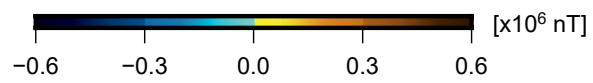

Figure C1. North polar views of the radial magnetic field component (a), its non-axisymetric part (b) and its non-dipolar part (c) at Earth's core surface. The red circles mark the intersection of the tangent cylinder with Earth's core-mantle boundary.

\section{Conflict of Interest}

The authors declare that they have no competing interests.

\section{Data Availability Statement}

All MESSENGER data used here are publicly available on NASA's Planetary Data System (PDS) https://doi. org/10.17189/1519745. Models and auxiliary files are available via https://doi.org/10.4121/14748345.v1.

\section{Acknowledgments}

The MESSENGER mission was supported by the NASA Discovery Program under contracts NAS5-97271 to The Johns Hopkins University Applied Physics Laboratory and NASW-00002 to the Carnegie Institution of Washington. This research was supported by the French Agence Nationale de la Rercherche, project MARMITE, contract 654 ANR13-BS05-0012, as well as by Centre National des Etudes Spatiales in the context of the BepiColombo MPO-MAG experiment. We are grateful for very constructive comments by anonymous reviewers. We thank L. Philpott and C. Johnson for sharing and discussing the magnetic activity index at Mercury and B. Anderson for the discussion of one of his Figures. Further, we would like to thank J. Wicht for suggesting to test for models with larger quadrupole-dipole ratio. All graphics were produced using open-source software, GMT (Wessel et al., 2013) and gnuplot.

\section{References}

Amit, H. (2014). Can downwelling at the top of the Earth's core be detected in the geomagnetic secular variation? Physics of the Earth and Planetary Interiors, 229, 110-121. https://doi.org/10.1016/j.pepi.2014.01.012

Amit, H., Aubert, J., \& Hulot, G. (2010). Stationary, oscillating or drifting mantle-driven geomagnetic flux patches? Journal of Geophysical Research: Solid Earth, 115(B7), B07108. https://doi.org/10.1029/2009JB006542

Amit, H., Korte, M., Aubert, J., Constable, C., \& Hulot, G. (2011). The time-dependence of intense archeomagnetic flux patches. Journal of Geophysical Research: Solid Earth, 116(B12), B12106. https://doi.org/10.1029/2011JB008538

Anderson, B. J., Acuña, M. H., Lohr, D. A., Scheifele, J., Raval, A., Korth, H., \& Slavin, J. A. (2007). The magnetometer instrument on MESSENGER. Space Science Reviews, 131(1), 417-450. https://doi.org/10.1007/s11214-007-9246-7

Anderson, B. J., Johnson, C. L., \& Korth, H. (2013). A magnetic disturbance index for Mercury's magnetic field derived from MESSENGER Magnetometer data. Geochemistry, Geophysics, Geosystems, 14, 3875-3886. https://doi.org/10.1002/ggge.20242

Anderson, B. J., Johnson, C. L., Korth, H., \& Philpott, L. C. (2018). Birkeland currents at mercury: Review and comparison with Earth In A. Keiling, O. Marghitu, \& M. Wheatland (Eds.), Electric currents in geospace and beyond (Vol. 235, pp. 279-302). https://doi. org/10.1002/9781119324522.ch17

Anderson, B. J., Johnson, C. L., Korth, H., Purucker, M. E., Winslow, R. M., Slavin, J. A., et al. (2011). The global magnetic field of mercury from MESSENGER orbital observations. Science, 333(6051), 1859-1862. https://doi.org/10.1126/science.1211001

Anderson, B. J., Johnson, C. L., Korth, H., Slavin, J. A., Winslow, R. M., Phillips, R. J., et al. (2014). Steady-state field-aligned currents at Mercury. Geophysical Research Letters, 41, 7444-7452. https://doi.org/10.1002/2014GL061677

Anderson, B. J., Johnson, C. L., Korth, H., Winslow, R. M., Borovsky, J. E., Purucker, M. E., et al. (2012). Low-degree structure in Mercury's planetary magnetic field. Journal of Geophysical Research: Planets, 117, E00L12. https://doi.org/10.1029/2012JE004159

Aubert, J. (2013). Flow throughout the Earth's core inverted from geomagnetic observations and numerical dynamo models. Geophysical Journal International, 192(2), 537-556. https://doi.org/10.1093/gji/ggs051

Backus, G., Parker, R., \& Constable, C. (1996). Foundations of geomagnetism. Cambridge University Press.

Bloxham, J., \& Gubbins, D. (1987). Thermal core-mantle interactions. Nature, 325, 511-513. https://doi.org/10.1038/325511a0

Bloxham, J., Gubbins, D., \& Jackson, A. (1989). Geomagnetic secular variation. Philosophical Transactions of the Royal Society of London-A, $329,415-502$

Buffett, B. A., King, E. M., \& Matsui, H. (2014). A physical interpretation of stochastic models for fluctuations in the Earth's dipole field. Geophysical Journal International, 198(1), 597-608. https://doi.org/10.1093/gji/ggu153

Busse, F. H. (1975). A model of the geodynamo. Geophysical Journal International, 42, 437-459. https://doi.org/10.1111/j.1365-246X.1975. tb05871.x 
Cao, H., Aurnou, J. M., Wicht, J., Dietrich, W., Soderlund, K. M., \& Russell, C. T. (2014). A dynamo explanation for Mercury's anomalous magnetic field. Geophysical Research Letters, 41(12), 4127-4134. https://doi.org/10.1002/2014GL060196

Cao, H., Yadav, R. K., \& Aurnou, J. M. (2018). Geomagnetic polar minima do not arise from steady meridional circulation. Proceedings of the National Academy of Sciences, 115(44), 11186-11191. https://doi.org/10.1073/pnas.1717454115

Christensen, U. (2006). A deep dynamo generating Mercury's magnetic field. Nature, 444, 1056-1058. https://doi.org/10.1038/nature05342

Christensen, U., Olson, P., \& Glatzmaier, G. A. (1998). A dynamo model interpretation of geomagnetic field structures. Geophysical Research Letters, 25(10), 1565-1568. https://doi.org/10.1029/98GL00911

Christensen, U., \& Wicht, J. (2008). Models of magnetic field generation in partly stable planetary cores: Applications to Mercury and Saturn. Icarus, 196, 16-34. https://doi.org/10.1016/j.icarus.2008.02.013

Christensen, U. R. (2018). Geodynamo models with a stable layer and heterogeneous heat flow at the top of the core. Geophysical Journal International, 215(2), 1338-1351. https://doi.org/10.1093/gji/ggy352

Christensen, U. R., Aubert, J., \& Hulot, G. (2010). Conditions for Earth-like geodynamo models. Earth and Planetary Science Letters, 296(3-4), 487-496. https://doi.org/10.1016/j.eps1.2010.06.009

Connerney, J. E. P., \& Ness, N. F. (1988). Mercury's magnetic field and interior. In Mercury (pp. 494-513). university of arizona press.

Dumberry, M., \& Rivoldini, A. (2015). Mercury's inner core size and core-crystallization regime. Icarus, 248, 254-268. https://doi.org/10.1016/j. icarus.2014.10.038

Finlay, C. C., Olsen, N., Kotsiaros, S., Gillet, N., \& Tøffner-Clausen, L. (2016). Recent geomagnetic secular variation from Swarm and ground observatories as estimated in the CHAOS-6 geomagnetic field model. Earth, Planets and Space, 68, 112. https://doi.org/10.1186/ s40623-016-0486-1

Gastine, T., Aubert, J., \& Fournier, A. (2020). Dynamo-based limit to the extent of a stable layer atop Earth's core. Geophysical Journal International, 222(2), 1433-1448. https://doi.org/10.1093/gji/ggaa250

Genova, A., Goossens, S., Mazarico, E., Lemoine, F. G., Neumann, G. A., Kuang, W., et al. (2019). Geodetic evidence that mercury has a solid inner core. Geophysical Research Letters, 46(7), 3625-3633. https://doi.org/10.1029/2018GL081135

Gubbins, D. (1975). Can the Earth's magnetic field be sustained by core oscillations? Geophysical Research Letters, 2, 409-412. https://doi. org/10.1029/g1002i009p00409

Gubbins, D. (1983). Geomagnetic field analysis-I. Stochastic inversion. Geophysical Journal of the Royal Astronomical Society, 73, 641-652. https://doi.org/10.1111/j.1365-246x.1983.tb03336.x

Gubbins, D., \& Bloxham, J. (1987). Morphology of the geomagnetic field and implications for the geodynamo. Nature, 325, 509-511. https:// doi.org/10.1038/325509a0

Hauck, S. A., Margot, J.-L., Solomon, S. C., Phillips, R. J., Johnson, C. L., Lemoine, F. G., et al. (2013). The curious case of Mercury's internal structure. Journal of Geophysical Research: Planets, 118(6), 1204-1220. https://doi.org/10.1002/jgre.20091

Holme, R., \& Bloxham, J. (1996). The magnetic fields of Uranus and Neptune: Methods and models. Journal of Geophysical Research, 101, 2177-2200. https://doi.org/10.1029/95je03437

Irving, J. C. E., Cottaar, S., \& Lekić, V. (2018). Seismically determined elastic parameters for Earth's outer core. Science Advances, 4(6), eaar2538. https://doi.org/10.1126/sciadv.aar2538

Jackson, A., Jonkers, A. R. T., \& Walker, M. R. (2000). Four centuries of geomagnetic secular variation from historical records. Philosophical Transactions of the Royal Society of London-A, 358, 957-990. https://doi.org/10.1098/rsta.2000.0569

Jackson, D. D. (1979). The use of a priori data to resolve nonuniqueness in linear inversion. Geophysical Journal of the Royal Astronomical Society, 57, 137-157. https://doi.org/10.1111/j.1365-246x.1979.tb03777.x

Jault, D. (2008). Axial invariance of rapidly varying diffusionless motions in the Earth's core interior. Physics of the Earth and Planetary Interiors, 166, 67-76. https://doi.org/10.1016/j.pepi.2007.11.001

Johnson, C. L., Purucker, M. E., Korth, H., Anderson, B. J., Winslow, R. M., Al Asad, M. M. H., et al. (2012). MESSENGER observations of Mercury's magnetic field structure. Journal of Geophysical Research: Planets, 117, E00L14. https://doi.org/10.1029/2012JE004217

Kaneshima, S. (2018). Array analyses of SmKS waves and the stratification of Earth's outermost core. Physics of the Earth and Planetary Interiors, 276, 234-246. https://doi.org/10.1016/j.pepi.2017.03.006

Konôpková, Z., McWilliams, R. S., Gómez-Pérez, N., \& Goncharov, A. F. (2016). Direct measurement of thermal conductivity in solid iron at planetary core conditions. Nature, 534(7605), 99-101. https://doi.org/10.1038/nature18009

Lesur, V., Wardinski, I., Rother, M., \& Mandea, M. (2008). GRIMM-the GFZ reference internal magnetic model based on vector satellite and observatory data. Geophysical Journal International, 173, 382-394. https://doi.org/10.1111/j.1365-246X.2008.03724.x

Lesur, V., Whaler, K. A., \& Wardinski, I. (2015). Are geomagnetic data consistent with stably stratified flow at the core-mantle boundary? Geophysical Journal International, 201, 929-946. https://doi.org/10.1093/gji/ggv031

Lhuillier, F., Fournier, A., Hulot, G., \& Aubert, J. (2011). The geomagnetic secular-variation timescale in observations and numerical dynamo models. Geophysical Research Letters, 38, 9306. https://doi.org/10.1029/2011GL047356

Long, R. S., Mound, J. E., Davies, C. J., \& Tobias, S. M. (2020). Scaling behaviour in spherical shell rotating convection with fixed-flux thermal boundary conditions. Journal of Fluid Mechanics, 889, A7. https://doi.org/10.1017/jfm.2020.67

Lowes, F. J. (1966). Mean-square values on sphere of spherical harmonic vector fields. Journal of Geophysical Research, 71, 2179. https://doi. org/10.1029/jz071i008p02179

Manglik, A., Wicht, J., \& Christensen, U. R. (2010). A dynamo model with double diffusive convection for Mercury's core. Earth and Planetary Science Letters, 289(3-4), 619-628. https://doi.org/10.1016/j.eps1.2009.12.007

Margot, J.-L., Hauck, I., Steven, A., Mazarico, E., Padovan, S., \& Peale, S. J. (2018). Mercury's internal structure. arXiv e-prints. arXiv:1806.02024.

Mauersberger, P. (1956). Das Mittel der Energiedichte des geomagnetischen Hauptfeldes an der Erdoberfläche und seine säkulare Änderung. Gerlands Beiträge zur Geophysik, 65, 207-215.

Mound, J., Davies, C., Rost, S., \& Aurnou, J. (2019). Regional stratification at the top of Earth's core due to core-mantle boundary heat flux variations. Nature Geoscience, 12(7), 575-580. https://doi.org/10.1038/s41561-019-0381-z

Ness, N. F. (1979). The magnetic field of Mercury. Physics of the Earth and Planetary Interiors, 20, 209-217. https://doi. org/10.1016/0031-9201(79)90044-X

Ness, N. F., Behannon, K. W., Lepping, R. P., Whang, Y. C., \& Schatten, K. H. (1974). Magnetic field observations near mercury: Preliminary results from Mariner 10. Science, 185, 151-160. https://doi.org/10.1126/science.185.4146.151

Oliveira, J. S., Hood, L. L., \& Langlais, B. (2019). Constraining the early history of mercury and its core dynamo by studying the crustal magnetic field. Journal of Geophysical Research: Planets, 124(9), 2382-2396. https://doi.org/10.1029/2019JE005938 
Oliveira, J. S., Langlais, B., Pais, M. A., \& Amit, H. (2015). A modified equivalent source dipole method to model partially distributed magnetic field measurements, with application to Mercury. Journal of Geophysical Research: Planets, 120, 1075-1094. https://doi. org/10.1002/2014JE004734

Olsen, N., Lühr, H., Sabaka, T. J., Mandea, M., Rother, M., Tøffner-Clausen, L., \& Choi, S. (2006). CHAOS-a model of the Earth's magnetic field derived from CHAMP, Ørsted, and SAC-C magnetic satellite data. Geophysical Journal International, 166, 67-75. https://doi. org/10.1111/j.1365-246X.2006.02959.x

Olson, P., \& Aurnou, J. (1999). A polar vortex in the Earth's core. Nature, 402(6758), 170-173. https://doi.org/10.1038/46017

Olson, P., Christensen, U., \& Glatzmaier, G. A. (1999). Numerical modeling of the geodynamo: Mechanisms of field generation and equilibration. Journal of Geophysical Research, 104, 10383-10404. https://doi.org/10.1029/1999JB900013

Olson, P., Landeau, M., \& Hirsh, B. H. (2017). Laboratory experiments on rain-driven convection: Implications for planetary dynamos. Earth and Planetary Science Letters, 457, 403-411. https://doi.org/10.1016/j.eps1.2016.10.015

Olson, P., Landeau, M., \& Reynolds, E. (2018). Outer core stratification from the high latitude structure of the geomagnetic field. Frontiers in Earth Science, 6, 140. https://doi.org/10.3389/feart.2018.00140

Panovska, S., Korte, M., \& Constable, C. G. (2019). One hundred thousand years of geomagnetic field evolution. Reviews of Geophysics, 57(4), 1289-1337. https://doi.org/10.1029/2019RG000656

Peale, S. J., Margot, J.-L., Hauck, S. A., \& Solomon, S. C. (2016). Consequences of a solid inner core on Mercury's spin configuration. Icarus, 264, 443-455. https://doi.org/10.1016/j.icarus.2015.09.024

Peña, D., Amit, H., \& Pinheiro, K. J. (2016). Magnetic field stretching at the top of the shell of numerical dynamos. Earth, Planets and Space, 68(1), 78. https://doi.org/10.1186/s40623-016-0453-x

Philpott, L. C., Johnson, C. L., Anderson, B. J., \& Winslow, R. M. (2020). The shape of Mercury's magnetopause: The picture from MESSENGER magnetometer observations and future prospects for BepiColombo. Journal of Geophysical Research: Space Physics, 125(5), e27544. https://doi.org/10.1029/2019JA027544

Poh, G., Slavin, J. A., Jia, X., Raines, J. M., Imber, S. M., Sun, W.-J., et al. (2017). Mercury's cross-tail current sheet: Structure, X-line location and stress balance. Geophysical Research Letters, 44(2), 678-686. https://doi.org/10.1002/2016GL071612

Pozzo, M., Davies, C., Gubbins, D., \& Alfè, D. (2012). Thermal and electrical conductivity of iron at Earth's core conditions. Nature, 485(7398), 355-358. https://doi.org/10.1038/nature11031

Shure, L., Parker, R. L., \& Backus, G. E. (1982). Harmonic splines for geomagnetic modelling. Physics of the Earth and Planetary Interiors, 28, 215-229. https://doi.org/10.1016/0031-9201(82)90003-6

Smith, D. E., Zuber, M. T., Phillips, R. J., Solomon, S. C., Hauck, S. A., Lemoine, F. G., et al. (2012). Gravity field and internal structure of mercury from MESSENGER. Science, 336(6078), 214-217. https://doi.org/10.1126/science.1218809

Stanley, S. (2010). A dynamo model for axisymmetrizing Saturn's magnetic field. Geophysical Research Letters, 37(5), L05201. https://doi. org/10.1029/2009GL041752

Stanley, S., Bloxham, J., Hutchison, W. E., \& Zuber, M. T. (2005). Thin shell dynamo models consistent with Mercury's weak observed magnetic field. Earth and Planetary Science Letters, 234, 27-38. https://doi.org/10.1016/j.eps1.2005.02.040

Stanley, S., \& Mohammadi, A. (2008). Effects of an outer thin stably stratified layer on planetary dynamos. Physics of the Earth and Planetary Interiors, 168(3-4), 179-190. https://doi.org/10.1016/j.pepi.2008.06.016

Stevenson, D. J. (1982). Reducing the non-axisymmetry of a planetary dynamo and an application to Saturn. Geophysical \& Astrophysical Fluid Dynamics, 2l(1), 113-127. https://doi.org/10.1080/03091928208209008

Takahashi, F., Shimizu, H., \& Tsunakawa, H. (2019). Mercury's anomalous magnetic field caused by a symmetry-breaking self-regulating dynamo. Nature Communications, 10, 208. https://doi.org/10.1038/s41467-018-08213-7

Tarantola, A. (1987). Inverse problem theory. Methods for data fitting and model parameter estimation. Elsevier.

Thébault, E., Finlay, C. C., Alken, P., Beggan, C. D., Canet, E., Chulliat, A., et al. (2015). Evaluation of candidate geomagnetic field models for IGRF-12. Earth, Planets and Space, 67, 112. https://doi.org/10.1186/s40623-015-0273-4

Thébault, E., Finlay, C. C., Beggan, C. D., Alken, P., Aubert, J., Barrois, O., et al. (2015). International geomagnetic reference field: The 12th generation. Earth, Planets and Space, 67, 79. https://doi.org/10.1186/s40623-015-0228-9

Thébault, E., Langlais, B., Oliveira, J. S., Amit, H., \& Leclercq, L. (2018). A time-averaged regional model of the Hermean magnetic field. Physics of the Earth and Planetary Interiors, 276, 93-105. https://doi.org/10.1016/j.pepi.2017.07.001

Tian, Z., Zuber, M. T., \& Stanley, S. (2015). Magnetic field modeling for Mercury using dynamo models with a stable layer and laterally variable heat flux. Icarus, 260, 263-268. https://doi.org/10.1016/j.icarus.2015.07.019

Uno, H., Johnson, C. L., Anderson, B. J., Korth, H., \& Solomon, S. C. (2009). Modeling Mercury's internal magnetic field with smooth inversions. Earth and Planetary Science Letters, 285, 328-339. https://doi.org/10.1016/j.epsl.2009.02.032

Van Hoolst, T., Rivoldini, A., Baland, R.-M., \& Yseboodt, M. (2012). The effect of tides and an inner core on the forced longitudinal libration of Mercury. Earth and Planetary Science Letters, 333, 83-90. https://doi.org/10.1016/j.epsl.2012.04.014

Vilim, R., Stanley, S., \& Hauck, S. A. (2010). Iron snow zones as a mechanism for generating Mercury's weak observed magnetic field. Journal of Geophysical Research: Planets, 115(E11), E11003. https://doi.org/10.1029/2009JE003528

Wardinski, I., Langlais, B., \& Thébault, E. (2019). Correlated time-varying magnetic fields and the core size of mercury. Journal of Geophysical Research: Planets, 124(8), 2178-2197. https://doi.org/10.1029/2018JE005835

Wessel, P., Smith, W. H. F., Scharroo, R., Luis, J., \& Wobbe, F. (2013). Generic mapping tools: Improved version released. Eos, Transactions American Geophysical Union, 94(45), 409-410. https://doi.org/10.1002/2013EO450001

Winslow, R. M., Anderson, B. J., Johnson, C. L., Slavin, J. A., Korth, H., Purucker, M. E., et al. (2013). Mercury's magnetopause and bow shock from MESSENGER Magnetometer observations. Journal of Geophysical Research, 118, 2213-2227. https://doi.org/10.1002/jgra.50237 